\title{
Expression Analysis of Sugarcane Aquaporin Genes under Water Deficit
}

\author{
Manassés Daniel da Silva, ${ }^{1}$ Roberta Lane de Oliveira Silva, ${ }^{1}$ \\ José Ribamar Costa Ferreira Neto, ${ }^{1}$ Ana Carolina Ribeiro Guimarães, ${ }^{2}$ \\ Daniela Truffi Veiga, ${ }^{2}$ Sabrina Moutinho Chabregas, ${ }^{2}$ William Lee Burnquist, ${ }^{2}$ \\ Günter Kahl, ${ }^{3}$ Ana Maria Benko-Iseppon, ${ }^{1}$ and Ederson Akio Kido ${ }^{1}$ \\ ${ }^{1}$ Department of Genetics, Federal University of Pernambuco (UFPE), 50670-901 Recife, PE, Brazil \\ ${ }^{2}$ Biotechnology Division, Sugarcane Technology Center (CTC), 13400-970 Piracicaba, SP, Brazil \\ ${ }^{3}$ Institute of Molecular Biosciences, Frankfurt University, 60438 Frankfurt am Main, Germany
}

Correspondence should be addressed to Ederson Akio Kido; kido.ufpe@gmail.com

Received 30 September 2013; Accepted 12 November 2013

Academic Editor: Shigenori Iwai

Copyright (c) 2013 Manassés Daniel da Silva et al. This is an open access article distributed under the Creative Commons Attribution License, which permits unrestricted use, distribution, and reproduction in any medium, provided the original work is properly cited.

\begin{abstract}
The present work is a pioneer study specifically addressing the aquaporin transcripts in sugarcane transcriptomes. Representatives of the four aquaporin subfamilies (PIP, TIP, SIP, and NIP), already described for higher plants, were identified. Forty-two distinct aquaporin isoforms were expressed in four HT-SuperSAGE libraries from sugarcane roots of drought-tolerant and -sensitive genotypes, respectively. At least 10 different potential aquaporin isoform targets and their respective unitags were considered to be promising for future studies and especially for the development of molecular markers for plant breeding. From those 10 isoforms, four (SoPIP2-4, SoPIP2-6, OsPIP2-4, and SsPIP1-1) showed distinct responses towards drought, with divergent expressions between the bulks from tolerant and sensitive genotypes, when they were compared under normal and stress conditions. Two targets (SsPIP11 and SoPIP1-3/PIP1-4) were selected for validation via RT-qPCR and their expression patterns as detected by HT-SuperSAGE were confirmed. The employed validation strategy revealed that different genotypes share the same tolerant or sensitive phenotype, respectively, but may use different routes for stress acclimation, indicating the aquaporin transcription in sugarcane to be potentially genotype-specific.
\end{abstract}

\section{Introduction}

Sugarcane (Saccharum spp.) is a valuable crop once it accumulates high levels of sucrose in the stems [1, 2]. In 2011, the twenty largest sugarcane producers generated about 1.7 billion tons of sucrose worldwide, valued about 52.5 billion dollars [3]. However, abiotic stresses can reduce the potential yield of these cultivated plants by $70 \%$, with drought being the most dangerous one [4]. Water deficit, and its influence onto a variable number of morphological and functional characters in plants, eventually becomes one of the main obstacles to sustainable agricultural production worldwide [5].

The reduction of the water content in a plant cell provokes a complex network of molecular responses, involving stress perception, signal transmission in a transduction cascade and physiological, cellular, and morphological changes [6], including stomatal closure, suppression of cell growth and photosynthesis, and activation of cellular respiration. Plants under drought still respond to it and adapt by accumulating specific osmolytes and proteins for stress tolerance [7].

Genes expressed during drought can be classified into two functional groups. The first group encodes proteins that increase plant tolerance to stress, such as water channels proteins (aquaporins), proteases, and detoxification enzymes, all having a protective function. To this group belong enzymes catalyzing the biosynthesis of osmolytes, like derivatives of amino acids, sugars and various LEA (LateEmbryogenesis-Abundant) proteins. The second group of 
genes encodes various proteins, such as transcription factors, kinases, phosphatases, and enzymes involved in regulatory pathways, as phospholipid metabolism and ABA biosynthesis [7]. The aquaporins or MIPs (Major Intrinsic Proteins) are proteins assembling into water channels of cell membrane and facilitate osmosis for rapid bidirectional transport of water [8]. Besides, these proteins are also involved in many plant metabolic processes, including acquisition of nutrients, cell growth, carbon fixation, cell signaling, and various stress responses $[9,10]$. The aquaporins also allow permeation of small molecules such as glycerol [11], urea [12] CO2 [13], ammonia [14], boric acid [15], H2O2 [16], and even arsenic [17]. According to the phylogenetic analysis of Johanson and Gustavsson [18], plant aquaporins are classified into four main subfamilies, widely distributed among higher plants: PIPs (plasma membrane intrinsic proteins), TIPs (tonoplast intrinsic proteins), SIPs (small basic intrinsic proteins), and NIPs (26 kDa intrinsic proteins). The aquaporins are presently and extensively studied, since their importance spans from animal [19] and human physiology [20] to osmoadaptation of microorganisms [21] and vegetables [22, 23]. The transcripts encoding sugarcane aquaporins have only marginally been described, despite their significant physiological influence and participation in several processes during plant growth and acclimation against biotic and abiotic stresses $[24,25]$.

The present study is a first attempt to derive expression markers (functional molecular markers) from HTSuperSAGE transcriptional profiles in contrasting sugarcane genotypes, in particular addressing specific sugarcane aquaporins, with the aim of better understanding the molecular processes occurring during drought response of the plant. HT-SuperSAGE, among all the genome-wide transcriptome profiling techniques was chosen for its efficiency to generate highly reliable transcription profiles. The increase in the size of the tag to $26 \mathrm{bp}$, the characteristic of SuperSAGE, drastically improves the annotation of the tag to the corresponding gene [28], allowing to establish genome-wide gene expression profiles of two or more species in one sample (e.g., hostparasite interactions $[29,30])$. Besides, SuperSAGE combined high-throughput next-generation sequencing [31, 32], designated DeepSuperSAGE or HT-SuperSAGE, provides even more informations (three to four orders of magnitude) at relatively low cost compared to traditional Sanger sequencing.

\section{Methodology}

2.1. Unitags Annotation, GO Categorization of ESTs, and Aquaporin Isoforms Identification. Bioinformatics analyses covered the 8,787,315 tags (26 bp) described by Kido et al. [33] from four root HT-SuperSAGE libraries [SD24T (the bulk of the tolerant genotypes CTC6, CTC15, SP83-2847, SP83-5073, under stress ( $24 \mathrm{~h}$ of continuous dehydration), totalizing 2,542,552 tags); SDTC (the tolerant bulk with daily irrigation, comprising 1,909,543 tags); SD24S (the sensitive bulk of stressed genotypes CTC9, CTC13, SP90-3414 and SP901638, with 2,170,998 tags) and SDSC (the bulk of sensitive genotypes without stress, covering 2,164,222 tags)]. After singlet exclusion (tags sequenced only once per library), the unique tags (unitags) were classified as up- (UR) or down-regulated (DR), based on the Audic and Claverie test $(P<0.05$; [34]), using the DiscoverySpace 4.0 software [35]. The unitag frequencies normalized to a million per library allowed the evaluation of the unitag expression modulation by fold change values (FC) comparing two frequencies. The unitags were aligned by BLASTn with expressed sequence tags (ESTs) from nine public databases, comprising sugarcane ESTs from NCBI (http://www.ncbi.nlm.nih.gov/nucest), and grass ESTs (Poaceae family) from Gene Index (http:// compbio.dfci.harvard.edu/tgi/plant.html), including Saccharum officinarum (SoGI 3.0), Sorghum bicolor (SbGI 9.0), Zea mays (ZmGI 19.0), Oryza sativa (OsGI 18.0), Panicum virgatum (PaviGI 1.0), Triticum aestivum (TaGI 12.0), Hordeum vulgare (HvGI 11.0), and Festuca arundinacea (FaGI $3.0)$. Only BLASTn alignments (e value $<0.0001)$ with scores 42 to 52 (100\% identity), plus/plus orientation and a preserved 5 'CATG were accepted, and the best tag - hit was selected prioritizing sugarcane sequences or sequences from closely related species with adequate annotation. ESTs anchoring unitags were then categorized via Gene Ontology (GO; http://www.geneontology.org/GO.doc.shtml), using the Blast2GO tool [36].

Potential ESTs from the MIP gene superfamily were identified using the keywords "aquaporin," "major intrinsic protein," "PIP," “TIP," “PIN," “SIP," “plasma membrane intrinsic protein" "tonoplast intrinsic protein," "nodulin-26like intrinsic protein" and "small basic intrinsic protein" in the EST annotations, or "water transporter" in the GO terms. These ESTs were classified into the plant aquaporin subfamilies (TIP, NIP, SIP, PIP) and analyzed with the NCBI Conserved Domain Search tool (http://www.ncbi.nlm.nih .gov/Structure/cdd/wrpsb.cgi) in an effort to confirm their conserved domains. Also ESTs were BLASTx aligned with proteins sequences from the UniProtKB/Swiss-Prot database (http://www.uniprot.org/help/uniprotkb), trying to confirm the isoform identity by using curated sequences ( $e$-value cutoff $\left.\mathrm{e}^{-10}\right)$.

2.2. Comparative and Phylogenetic Analysis of the Putative Aquaporin Isoforms Based on Unitag Expressions. The predicted peptides from ESTs related to the tags after translation with the ORF finder tool (http://www.ncbi.nlm.nih.gov/ projects/gorf/) and BLASTp analysis were subsequently aligned via Clustal W [37]. A dendrogram of the aligned sequences was generated using the MEGA v.5.2.1 software [27], according to the following parameters: Neighbor Joining tree method, pairwise deletion option, and 1000 bootstrap replicates. For a better assignment of the isoforms into the aquaporin subfamilies, $15 \mathrm{~A}$. thaliana aquaporin protein sequences were included in addition to the predicted proteins. Also, two sequences served as outgroups, one from humans and one from Yersinia pseudotuberculosis. In addition to the phylogenic tree, a heat map was established based on fold changes of the unitags responding to the applied stress. 
2.3. cDNA Synthesis, Primer Design, and RT-qPCR Analysis. The RNA of each genotype sample constituting the bulks (tolerant stressed, tolerant control, sensitive stressed, and sensitive control) was isolated from sugarcane roots using the RNAeasy Plus Micro Kit (Qiagen) and DNase treatment. The cDNA synthesis reaction was performed with the SuperScriptTM First Strand kit Synthesis System for RT-PCR (Invitrogen) according to the manufacturer's protocol, using $1 \mathrm{~g}$ of RNA quantified by the Qubit fluorometer (Invitrogen). Primer pairs were designed from ESTs anchoring unitags, tolerating a maximum of one mismatch, and using the default parameters of the Primer3 software [38] with some minor modifications [amplicon size: 70 to $200 \mathrm{bp}$; primer Tm: 40 to $60^{\circ} \mathrm{C}$; GC content: 45 to $55 \%$ ]. These ESTs anchored unitags differentially regulated in the tolerant and sensitive genotype bulks. Prior to the validation of unitags by RTqPCR, standard curves using a dilution series of the cDNA pool were made to calculate the gene-specific PCR efficiency and regression coefficient $\left(R^{2}\right)$ for each gene (Table 6). The RT-qPCR amplifications were performed on the LineGene 9660 model (Bioer), using SYBR Green detection. Each reaction mixture comprised $1 \mu \mathrm{L}$ of template cDNA (diluted 5 -fold), $5 \mu \mathrm{L}$ of HotStart-IT SYBR Green qPCR Master Mix $2 x$ (USB), $0.05 \mu \mathrm{L}$ of ROX, $1.95 \mu \mathrm{L}$ of water, and $1 \mu \mathrm{L}$ primer $(500 \mathrm{nM}$ each) to a final volume of $10 \mu \mathrm{L}$. The reactions were denatured at $95^{\circ} \mathrm{C}$ for $2 \mathrm{~min}$, followed by 40 cycles of $95^{\circ} \mathrm{C}$ for $15 \mathrm{~s}, 58^{\circ} \mathrm{C}$ for $30 \mathrm{~s}$, and $72^{\circ} \mathrm{C}$ for $15 \mathrm{~s}$ in $96-$ well reaction plates, with the detection of the fluorescence signal at the end of each extension step. The melting curves were analyzed at $65-95^{\circ} \mathrm{C}$ for $20 \mathrm{~min}$ after 40 cycles. Three biological replicates and three technical replicates were used for RT-qPCR analysis. The relative quantification data were analyzed with the $\operatorname{REST}^{\odot}$ v.2.0.13 software [26].

\section{Results and Discussion}

3.1. Aquaporin Data-Mining and Categorization of EST Anchoring Unitags. The universe of 8,787,315 tags (26 bp) generated from the four HT-SuperSAGE libraries presented 205,975 unique tags (unitags) [33], from which 289 anchored in 484 putative aquaporin ESTs, distributed in nine different databases (details in Table 1), totaling 1,579 BLASTn results with scores of 42 to 52 (100\% identity). This set of 484 ESTs anchoring unitags (Table 1) represented the basis for the analysis of transcript profiles based on the respective unitags.

The keyword ("aquaporin," "major intrinsic protein," "tonoplast intrinsic protein," "plasma membrane intrinsic protein," "small basic intrinsic protein," "nodulin-26-like intrinsic protein," "PIP," “TIP," "NIP," "SIP") searches in the EST annotation identified 1,347 ESTs, while the "water transport" GO expression identified 342 ESTs (Figure 1). The searches in the GO terms increased the aquaporin identifications by almost 15\%, representing 230 alignments of the total (1,579; Figure 1).

The unitag annotation efficiency relied on the used EST database. As mentioned by Kido et al. [39], Gene Index is a good source for unitag annotation, as it displays adequate gene or protein function descriptions. In the present case,

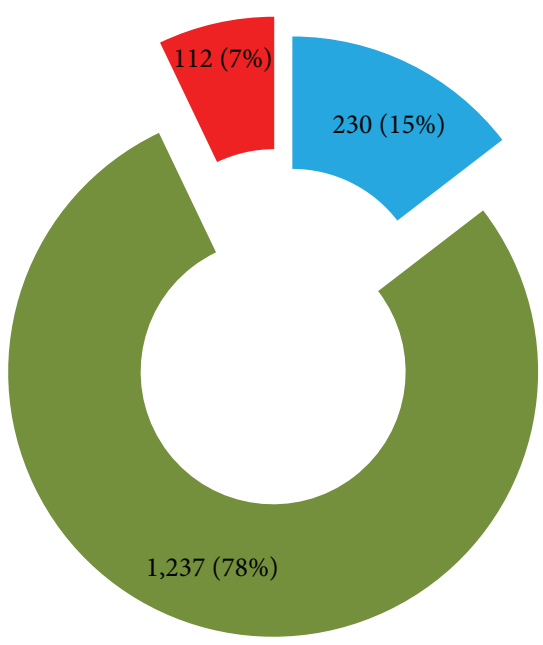

Without annotation, but with GO term

"water transport"

- With annotation, but without GO term "water transport"

With both annotation and GO term "water transport"

Figure 1: Percentage of HT-SuperSAGE unitags from sugarcane plants ( $24 \mathrm{~h}$ of continuous dehydration or regular daily irrigation) identified as potential aquaporins after keyword searches in the EST annotation ("aquaporin," "tonoplast intrinsic protein," "PIP," "TIP," "NIP," "SIP”) or in the GO terms ("water transport"). Total of unitags: 1,579 .

the SoGI (Saccharum officinarum L.) dataset representing 282,683 ESTs that resulted in 121,342 unique sequences [42,377 TC (Tentative Consensus clusters) plus 78,965 singletons) after clustering. This species took part in the sugarcane breeding programs [40, 41] performed around the world.

Almost all unitags related to the aquaporin annotations (260 of 289; Table 1) anchored in SoGI sequences, which exceeded those obtained with the partial dbEST dataset (http://www.ncbi.nlm.nih.gov/dbEST/dbEST_summary.

html) by almost six times. This dbEST is composed of 277,266 ESTs, mostly from Brazilian sugarcane hybrids (SUCESTFAPESP project) [42] (Table 1). This may mainly be due to the sizes of the SoGI sequences, as most alignments occurred in the CTs. Nevertheless, the partial dbEST dataset was the second best source for mapping unitags, but its real annotation power was affected by the nonadequate descriptions of the cDNAs (many "unknown" hits). As Sorghum bicolor is the most closely related diploid of S. officinarum [2], this species could contribute to the identification of aquaporin isoforms. However, after redundancy exclusion, only 19 unitags anchored in seven unique ESTs based on the best hits (Table 1). This poor performance may be explained by the low number of ESTs available in the SbGI dataset $(46,043)$, being the second smallest databank used (Table 1), in contrast with the high number of available sugarcane ESTs, reinforced by the high homology between sorghum and sugarcane. 
TABLE 1: EST databases used for unitag annotations from sugarcane HT-SuperSAGE libraries (tolerant/sensitive genotypes after $24 \mathrm{~h}$ of continuous dehydration or regular daily irrigation) and BLASTn nonredundant results.

\begin{tabular}{|c|c|c|c|c|c|c|c|c|c|c|c|c|}
\hline Species & Database & Annot.*/ESTs & PIP & TIP & NIP & SIP & $\begin{array}{c}\text { Total } \\
\text { BlastN } \\
\text { Align. }\end{array}$ & $\begin{array}{c}\text { Score } \\
52\end{array}$ & $\begin{array}{l}\text { Align. } \\
\text { (up to one } \\
\text { mismatch) }\end{array}$ & Unitags & $\begin{array}{l}\text { Unique } \\
\text { ESTs }^{+}\end{array}$ & $\begin{array}{c}\text { Unitags } \\
\text { with } \mathrm{GO}^{1}\end{array}$ \\
\hline Saccharum spp. & $\mathrm{dbEST}$ & $24 / 256,636$ & 0 & 0 & 0 & 0 & 154 & 41 & 105 & 43 & 34 & 23 \\
\hline Saccharum officinarum & SoGI & $265 / 121,342$ & 157 & 85 & 21 & 11 & 683 & 158 & 425 & 260 & 127 & 50 \\
\hline Sorghum bicolor & SbGI & $68 / 46,043$ & 34 & 23 & 5 & 6 & 74 & 11 & 63 & 19 & 7 & 74 \\
\hline Zea mays & ZmGI & $347 / 315,134$ & 142 & 154 & 35 & 19 & 49 & 7 & 29 & 13 & 15 & 20 \\
\hline Oryza sativa & OsGI & $283 / 201,220$ & 121 & 95 & 33 & 7 & 119 & 26 & 72 & 20 & 86 & 3 \\
\hline Panicum virgatum & PaviGI & $147 / 85,244$ & 58 & 45 & 28 & 6 & 174 & 31 & 116 & 22 & 57 & 0 \\
\hline Triticum aestivum & TaGI & $542 / 222,152$ & 138 & 128 & 32 & 14 & 253 & 20 & 197 & 23 & 123 & 5 \\
\hline Hordeum vulgare & HvGI & $110 / 83,101$ & 32 & 33 & 12 & 9 & 34 & 2 & 30 & 16 & 21 & 7 \\
\hline Festuca arundinacea & FaGI & $27 / 30,244$ & 5 & 1 & 1 & 1 & 39 & 10 & 27 & 17 & 14 & 5 \\
\hline Total & & $1,913 / 1,361,144$ & 687 & 564 & 167 & 73 & 1,579 & 306 & 1,064 & $289^{\#}$ & 484 & $45^{\#}$ \\
\hline
\end{tabular}

${ }^{*}$ Aquaporin, tonoplast intrinsic protein and major intrinsic protein, membrane integral protein (PIP, TIP, NIP e SIP); ${ }^{+}$number of nonredundant ESTs (putative aquaporins) anchoring unitags, "water transporter"; " number without redundancy among the nine databanks.

The unitags proved to be highly specific for aquaporins. A total of 263 unitags (91\% of 289) were associated with aquaporin isoforms (189 unitags anchored in just a single EST from a unique database); 19 unitags (7\%) were not isoformspecific but comprised the same subclass (PIP1 or PIP2) and only seven $(2 \%)$ were not specific to any subclass.

3.2. Comparative Analysis of the Putative Aquaporin Isoforms Identified by the Unitags. Regarding the total of 484 annotated aquaporin-ESTs anchoring unitags (Table 1), 470 of them $(97.10 \%)$ aligned with aquaporin-proteins isoforms with an $e$ value $<10^{-20}$ (BLASTx), from the UniProtKB/SwissProt database, a high-quality annotated databank. This fact confirmed the isoforms identities (data not shown). All of them represented 42 distinct isoforms belonging to the four subfamilies (PIP, TIP, SIP, and NIP) based on the ESTs annotations. The 42 isoforms and their respective number of unitags [considered UR, DR or n.s. $(P<0.05)$ ] in the two main HT-SuperSAGE libraries contrasts are shown in Table 2. According to this table, one unitag or more could be associated with a specific isoform. In some cases, two or more ESTs from one database present the same isoform annotation. The Gene Index databases used throughout this work minimized this situation due to the assembled TC (Tentative Consensus) clusters. Besides, unitags aligning more than one locus in the same EST could be resulted by partial NlaIII digestions. In an attempt to avoid this situation, it was performed double digestions. Additionally, this event could be resulted by sister-tags anchoring one specific EST and isoform. In this case, tags showing a single base substitution (sister-tags) were considered as two different unitags. On the other hand, alternative transcripts could anchor varied unitags. Also, specific isoforms could be mapped in several loci (in the same or in different chromosomes). In addition, Saccharum hybrids show complex genomes, as a result of polyploidy and aneuploidy events [40, 41]. Therefore, this diversity of unitags (UR and DR) associated to aquaporin isoforms could allow identify biotechnologically interesting candidates.

From the 71 unique ESTs involved in perfect unitagEST BlastN alignments (score 52; Table 1), 24 putative aquaporins showed ORFs with over 180 amino acids in size, and these sequences, together with MIP protein sequences from Arabidopsis thaliana, Homo sapiens, and Yersinia pseudotuberculosis were compared in a phenetic analysis. The resulting tree confirmed that putative aquaporins clearly divide into four major clusters, representing the PIP, TIP, SIP, and NIP subfamilies (Figure 2). This tree was consistent with a previous analysis of aquaporin phylogeny in higher plants [10, $18,43,44]$. As expected, the human HsAPQ1 isoform grouped with the PIP subfamily, since the human APQ1 subfamily was recently recognized to be phylogenetically more similar to the PIP subfamily than to other plant subfamilies [45]. Also, YpGIpF grouped with the NIP aquaporin subfamily. The YpGIpF isoform belongs to a MIP family related to the bacterial GlpF protein glycerol uptake facilitator, classically associated with aquaglyceroporins from NIP and APQ3 subfamilies [45]. Therefore, this tree, which is supported by the scientific literature, presents the 24 aquaporin isoforms identified by HT-SuperSAGE unitags expressed after $24 \mathrm{~h}$ of continuous dehydration stress. Moreover, considering only the nine isoforms identified from S. officinarum ESTs, this smaller set was also distributed across the four aquaporin subfamilies described for higher plants.

Additionally, the heat maps (Figure 2) revealed by the expression modulation of the unitags (FCs) in the tolerant or sensitive bulks (both with their respective unstressed controls) show that some PIP isoforms are divergently regulated in the bulks of genotypes. Thus, from the 12 PIP transcripts, eight were repressed in the tolerant genotypes under stress. At the same time, eight of those transcripts were induced in the sensitive bulk. Furthermore, the majority of the PIP transcripts showed divergent modulations (contrasting 


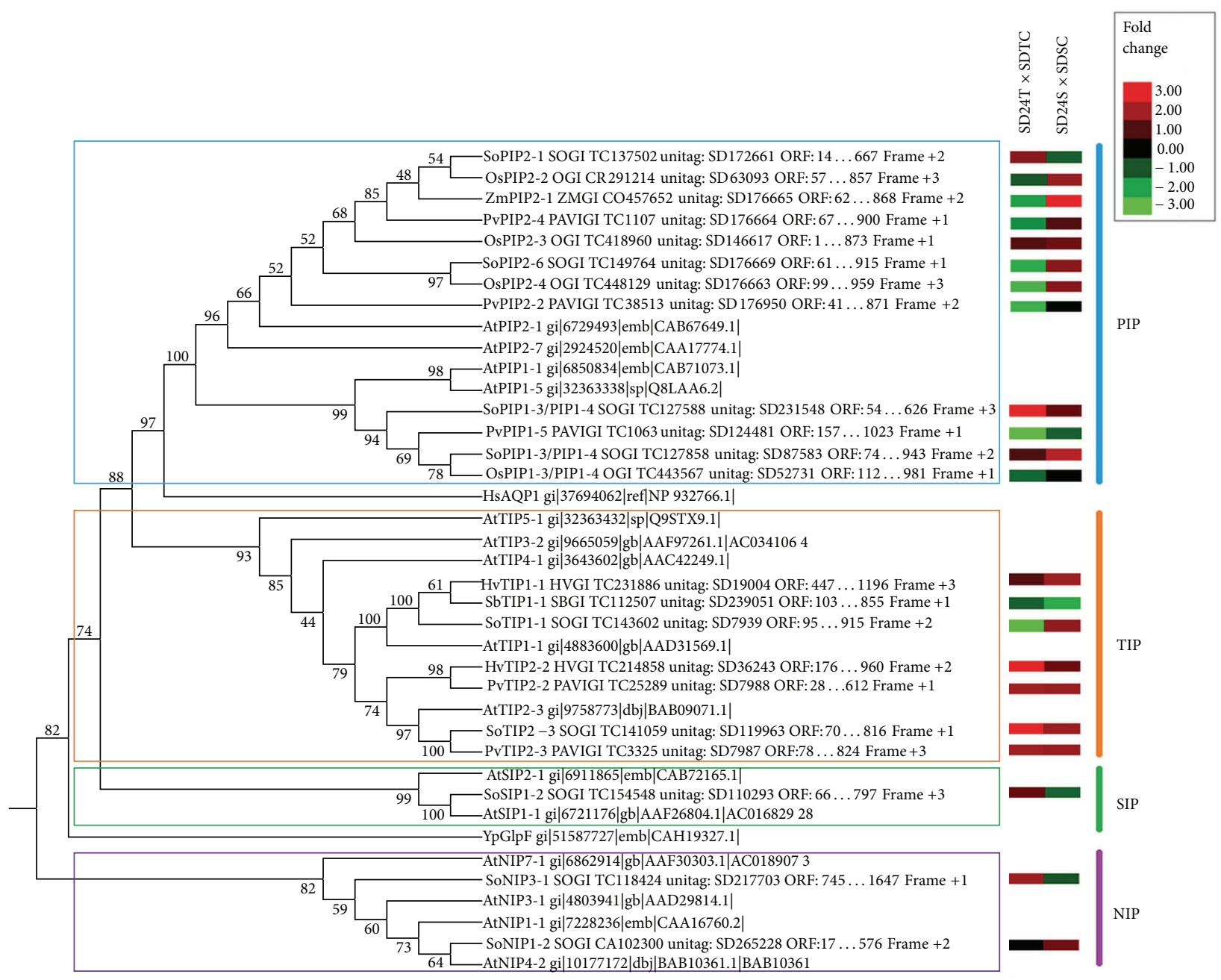

Figure 2: Neighbor Joining dendrogram (MEGA v.5.2.1 software [27]) and integrated heat map (bootstrap values of 1,000 replications), showing the phenetic grouping of 24 potential aquaporin amino acid sequences identified by HT-SuperSAGE unitags from sugarcane accessions after $24 \mathrm{~h}$ of continuous dehydration (with their respective EST and unitag identifiers), and aquaporins sequences of Arabidopsis thaliana, human and Yersinia pseudotuberculosis (all these labeled by an asterisk). Nomenclature: Isoforms are preceded by the abbreviated species name (At-Arabidopsis thaliana; Hs-Homo sapiens; Hv-Hordeum vulgare; Os-Oryza sativa; Pv-Panicum virgatum; Sb-Sorghum bicolor; So-Saccharum officinarum; Yp-Yersinia pseudotuberculosis; Zm-Zea mays).

results) when the response of both bulks of stressed genotypes is compared. Taken together, different genotypes may have developed different survival strategies.

On the other hand, the TIP transcripts similarly responded to the stress (comparing the modulation between both bulks of genotypes, Figure 2). Of the seven TIP subfamily isoforms studied (Figure 2), five were induced in both bulks of genotypes responding to stress, suggesting the participation of these isoforms in water transport. Finally, the only SIP subfamily representative studied here showed distinct regulation between the analyzed bulks, whereas the two NIP subfamily representatives distinctly responded: NIP3-1 was induced in the tolerant bulk and suppressed in its sensitive counterpart, while NIP3-2 was not modulated in the tolerant bulk, but was induced in the sensitive bulk (Figure 2). For these subfamilies, a larger amount of data is required for further analysis.
3.3. Transcriptional Profile of Putative Aquaporins Based on Unitags. The 30 most expressed unitags, based on their normalized frequencies (tpm) in the HT-SuperSAGE libraries, associated to the aquaporin subfamilies PIP (15), TIP (10), SIP (3) and NIP (2) are displayed in Table 3. According to Kjellbom et al. [46] many aquaporin genes are constitutively expressed, with a large number of transcripts (as presented in Table 3), while others are temporally and spatially regulated during plant development or stress responses, as is, for example, the case with unitag SD173276 (Table 3).

After necessary redundancy exclusion, we identified 42 potential aquaporin isoforms. The contribution of each aquaporin subfamily is presented in Figure 3. In each comparison (SD24T vs SDTC: 26; SD24S vs SDSC: 28; SD24T vs SD24S: 28; SDTC vs SDSC: 28 ), 26-28 isoforms were identified as being expressed in sugarcane roots after onset of stress $(24 \mathrm{~h}$ of continuous dehydration) or under normal daily irrigation 
TABLE 2: Putative sugarcane aquaporin isoforms (42) based on unitags of root dehydration $(24 \mathrm{~h})$ observed in the two main contrasts of HT-SuperSAGE libraries.

\begin{tabular}{|c|c|c|c|c|c|c|}
\hline \multirow{2}{*}{$\begin{array}{l}\text { Aquaporin } \\
\text { isoform }\end{array}$} & \multicolumn{3}{|c|}{ SD24T versus SDTC } & \multicolumn{3}{|c|}{ SD24S versus SDSC } \\
\hline & UR & $\mathrm{DR}$ & n.s. & UR & DR & n.s. \\
\hline SsPIP1-1 & 1 & - & 2 & - & 1 & 1 \\
\hline SoPIP1-2 & 1 & 2 & 3 & 2 & 1 & - \\
\hline$P v$ PIP1-2 & - & - & 1 & - & - & 1 \\
\hline SoPIP1-3/PIP1-4 & 3 & 24 & 26 & 7 & 7 & 25 \\
\hline OsPIP1-3/PIP1-4 & 1 & - & 2 & - & 1 & - \\
\hline SoPIP1-5 & 1 & 2 & 2 & 3 & - & 2 \\
\hline PvPIP1-5 & - & - & 2 & - & - & 1 \\
\hline SoPIP2-1 & 2 & 14 & 23 & 4 & 24 & 19 \\
\hline ZmPIP2-1 & - & 2 & 4 & 1 & - & 4 \\
\hline OsPIP2-1 & 1 & - & 2 & - & 1 & 2 \\
\hline PvPIP2-1 & - & 1 & 9 & 1 & - & 6 \\
\hline TaPIP2-1 & - & 2 & 4 & 1 & - & 2 \\
\hline OsPIP2-2 & - & - & 2 & - & - & 3 \\
\hline$P v$ PIP2-2 & - & 2 & 6 & 1 & - & 4 \\
\hline OsPIP2-3 & - & - & 1 & - & - & 1 \\
\hline ZmPIP2-3 & - & - & 1 & - & - & 1 \\
\hline SoPIP2-4 & 3 & 4 & 6 & 2 & 3 & 6 \\
\hline OsPIP2-4 & - & 2 & - & 1 & 2 & - \\
\hline$P v$ PIP2-4 & - & 4 & 2 & 1 & - & 5 \\
\hline FaPIP2-4 & - & 3 & 7 & 3 & - & 3 \\
\hline SoPIP2-5 & 1 & 1 & - & - & - & 2 \\
\hline TaPIP2-5 & - & 1 & 4 & 1 & - & 2 \\
\hline SoPIP2-6 & 2 & - & 2 & 2 & - & 2 \\
\hline TaPIP2-6 & - & 1 & 3 & 1 & - & 2 \\
\hline SsTIP1-1 & 4 & 9 & 14 & 6 & 5 & 18 \\
\hline SoTIP1-1 & 3 & 7 & 10 & 6 & 7 & 11 \\
\hline SbTIP1-1 & 1 & - & 1 & - & 1 & 1 \\
\hline ZmTIP1-1 & - & - & 1 & - & - & - \\
\hline$H v$ TIP1-1 & 1 & - & - & - & 1 & - \\
\hline TaTIP1-1 & - & 1 & 4 & - & 1 & 4 \\
\hline TaTIP1-2 & - & 1 & - & - & - & 1 \\
\hline SoTIP2-2 & 2 & 9 & 9 & 1 & 8 & 11 \\
\hline$H v \mathrm{TIP} 2-2$ & 1 & - & 1 & - & - & 1 \\
\hline$P v$ TIP2-2 & - & - & 2 & - & - & 2 \\
\hline SoTIP2-3 & 5 & 5 & 10 & 3 & 3 & 5 \\
\hline PvTIP2-3 & - & - & 3 & - & - & 3 \\
\hline SoTIP4-2 & - & - & 1 & - & - & 1 \\
\hline$P v$ NIP1-1 & - & 1 & - & - & 1 & 1 \\
\hline SoNIP1-2 & - & - & 1 & - & - & 1 \\
\hline SoNIP3-1 & 1 & 2 & 12 & - & 1 & 10 \\
\hline SoSIP1-1 & 1 & - & 2 & 1 & - & 1 \\
\hline SoSIP1-2 & 3 & 5 & 2 & 2 & 1 & 10 \\
\hline
\end{tabular}

Isoforms are preceded by the abbreviated species name (Fa: Festuca arundinacea; Hv: Hordeum vulgare; Os: Oryza sativa; Pv: Panicum virgatum; Sb: Sorghum bicolor; So: Saccharum officinarum; Ss: Saccharum spp.; Ta: Triticum aestivum; Zm: Zea mays). HT-SuperSAGE libraries: SD24T (bulk of tolerant genotypes under stress; SD24S (bulk of sensitive genotypes under stress); SDTC (control bulk of tolerant genotypes); SDSC (control bulk of sensitive genotypes). DR: down-regulated; UR: up-regulated. n.s.: not significant at $P$ $<0.05$.

conditions. This number of isoforms is close to that of other higher plants (31 aquaporin isoforms in maize [43, 47], 35 in A. thaliana [48], 39 in rice [47]), and more than twice the
TABLE 3: The 30 HT-SuperSAGE unitags most expressed and annotated as aquaporins from sugarcane libraries with contrasting genotypes under stress ( $24 \mathrm{~h}$ of continuous dehydration) or normal daily irrigation.

\begin{tabular}{|c|c|c|c|c|c|}
\hline \multirow{2}{*}{ Unitag id } & \multirow{2}{*}{ Aquaporin } & \multicolumn{4}{|c|}{ Tags per million (tpm) } \\
\hline & & SD24T & SDTC & SD24S & SDSC \\
\hline SD173282 & SoTIP2-2 & 1,096 & 3,784 & 1,643 & 1,816 \\
\hline SD231437 & SoPIP1-3/PIP1-4 & 819 & 1,551 & 1,140 & 990 \\
\hline SD87583 & SoPIP1-3/PIP1-4 & 964 & 956 & 1,186 & 520 \\
\hline SD119746 & SoTIP2-3 & 1,162 & 1,041 & 771 & 530 \\
\hline SD173276 & SoTIP2-2 & 564 & 1,879 & 496 & 0 \\
\hline SD182865 & SoTIP2-2 & 535 & 876 & 501 & 674 \\
\hline SD87593 & SoPIP1-3 /PIP1-4 & 579 & 601 & 750 & 377 \\
\hline SD80613 & SoTIP1-1 & 318 & 234 & 775 & 571 \\
\hline SD80612 & SoTIP1-1 & 437 & 393 & 423 & 453 \\
\hline SD250744 & SoPIP2-1 & 321 & 577 & 265 & 508 \\
\hline SD19004 & $H v$ TIP1-1 & 422 & 395 & 411 & 210 \\
\hline SD176669 & SoPIP2-6 & 275 & 591 & 310 & 183 \\
\hline SD243880 & SoPIP2-1 & 176 & 326 & 334 & 496 \\
\hline SD28080 & SoPIP2-4 & 406 & 329 & 224 & 312 \\
\hline SD176663 & SoPIP2-6 & 135 & 340 & 227 & 136 \\
\hline SD241279 & SoPIP1-5 & 151 & 184 & 257 & 107 \\
\hline SD84960 & SsTIP1-1 & 202 & 216 & 29 & 136 \\
\hline SD243849 & SoPIP2-1 & 108 & 123 & 113 & 40 \\
\hline SD54852 & SoPIP2-1 & 107 & 143 & 22 & 52 \\
\hline SD96918 & SoSIP1-2 & 106 & 66 & 96 & 49 \\
\hline SD96922 & SoSIP1-2 & 89 & 43 & 114 & 49 \\
\hline SD202395 & $P v$ NIP1-1 & 39 & 53 & 40 & 98 \\
\hline SD243867 & SoPIP2-1 & 37 & 42 & 79 & 43 \\
\hline SD87586 & SoPIP1-3/PIP1-4 & 31 & 54 & 28 & 55 \\
\hline SD250859 & SoPIP2-1 & 39 & 96 & 0 & 32 \\
\hline SD198883 & SoPIP2-5 & 39 & 27 & 30 & 37 \\
\hline SD21811 & SoSIP1-1 & 26 & 16 & 26 & 26 \\
\hline SD84958 & SsTIP1-1 & 29 & 15 & 9 & 17 \\
\hline SD217703 & SoNIP3-1 & 29 & 15 & 13 & 13 \\
\hline SD36243 & $H v$ TIP2-2 & 31 & 9 & 12 & 9 \\
\hline
\end{tabular}

Isoforms are preceded by the abbreviated species name (Hv: Hordeum vulgare; Pv: Panicum virgatum; So: Saccharum officinarum; Ss: Saccharum spp). SuperSAGE libraries: SD24T (bulk of tolerant genotypes under stress; SD24S (bulk of sensitive genotypes under stress); SDTC (control bulk of tolerant genotypes); SDSC (control bulk of sensitive genotypes).

amount predicted for vertebrates (11 to 13 isoforms) [49, 50]. The number of aquaporin isoforms in sugarcane may be even higher, since some isoforms respond only in specific tissues [51] or after - salinity [52], freezing [53], mycorrhization [54], light $[55,56]$, and cell growth stresses [10]. Therefore, the real number of aquaposin isoforms can only be estimated approximatively by whole genome sequencing. Since sugarcane has one of the most complex genomes of the plant kingdom, with a diploid number of chromosomes ranging from 100 to 130 as a result of aneuploidy and polyploidy events [41], this approach would require significant efforts and investments.

The most transcribed 19 aquaporin unitags belonged to the PIP and TIP subfamilies (Table 3), which matches a report by Alexandersson et al. [51], who analyzed the transcriptional 


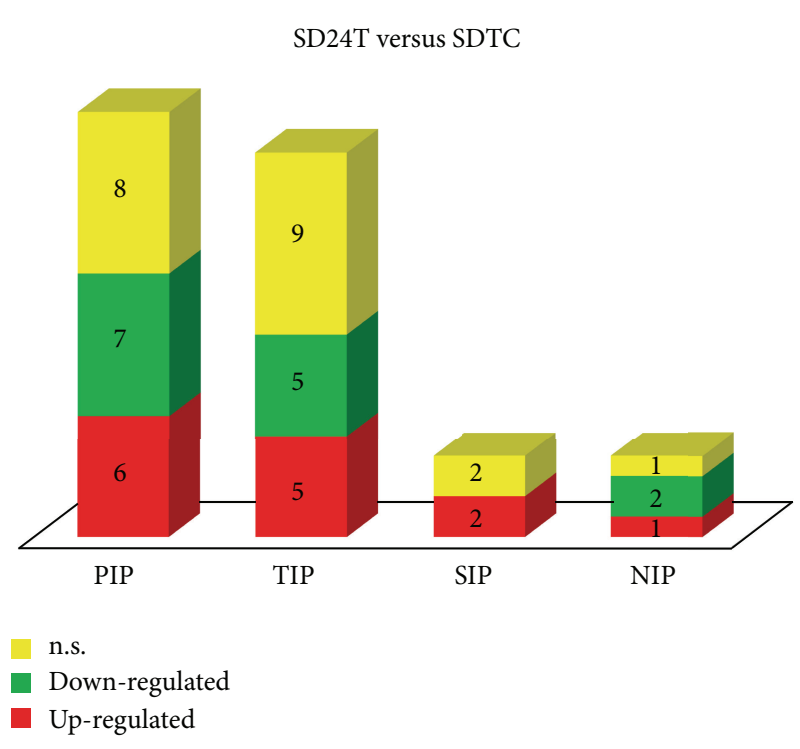

(a)

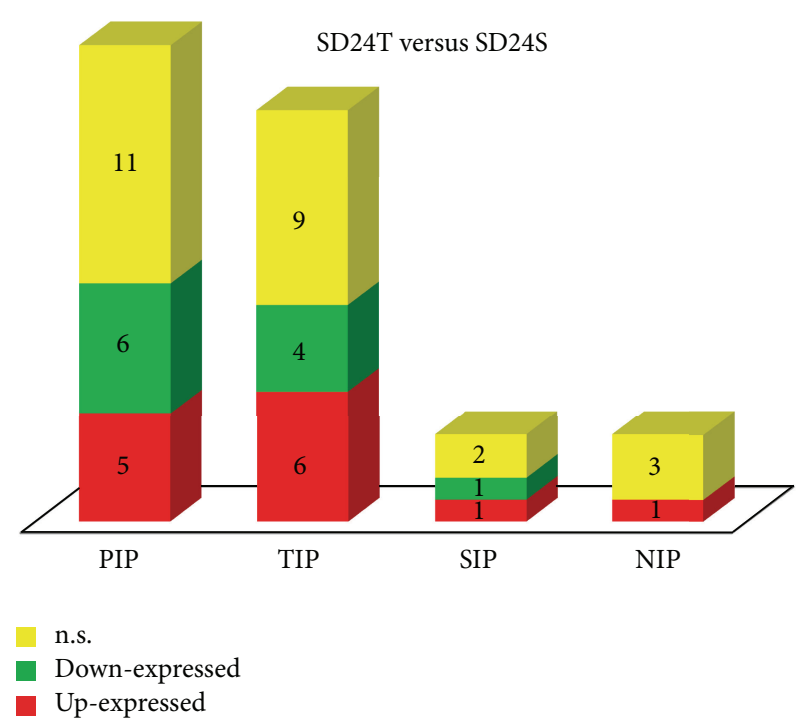

(c)

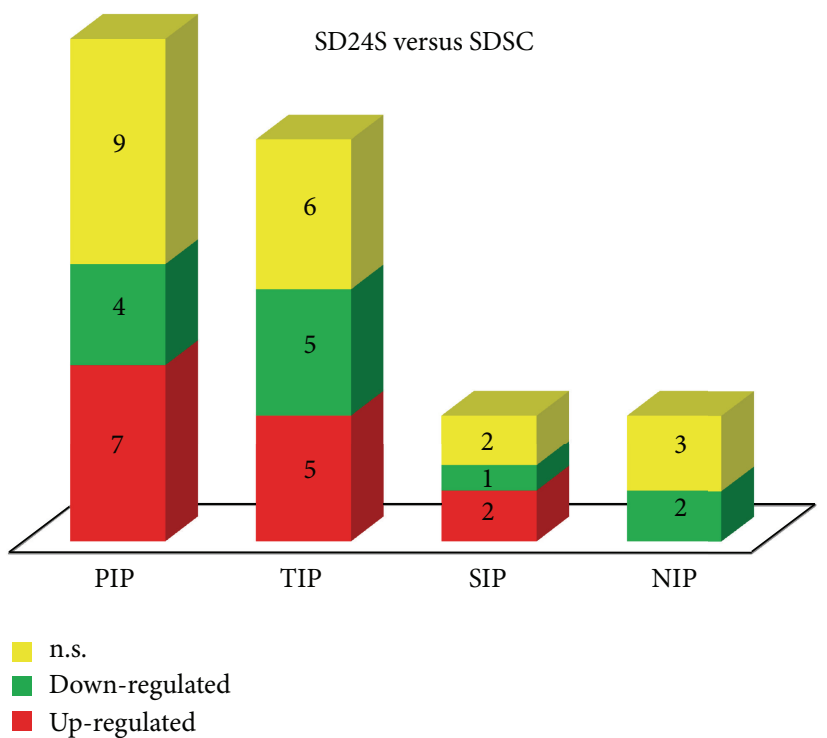

(b)

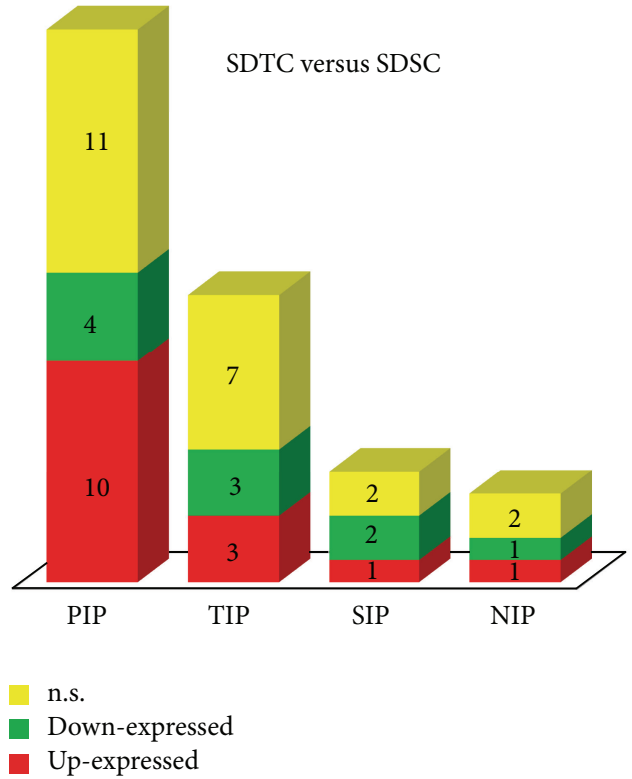

(d)

FIGURE 3: Representation of aquaporin subfamilies expressed in sugarcane after $24 \mathrm{~h}$ of continuous dehydration ((a), (b), and (c)) and during normal irrigation (d), involving bulks of genotypes tolerant and sensitive to stress. HT-SuperSAGE libraries: SD24T (bulk of tolerant genotypes under stress; SD24S (bulk of sensitive genotypes under stress); SDTC (control bulk of tolerant genotypes); SDSC (control bulk of sensitive genotypes).

profile of 35 Arabidopsis aquaporins in three different tissues (roots, leaves, and flowers) during water deficit stress (watering suppression). These authors concluded that in all the studied tissues, the PIP, and TIP aquaporins showed higher expression levels, whereas NIPs aquaporins exhibited particularly low transcriptional levels under stress. Zhu et al. [52] also confirmed a lower amount of NIP and SIP in corn under controlled conditions (continuously aerated hydroponic medium, and parameters described by Gibeaut et al. [57]), as compared to the PIP and TIP, which could be related to the aquaporin transport specificity [58]. NIPs are related to the transport of small solutes [31], whereas the physiological functions of SIPs, in addition to water transport [59], still remain unclear. Otherwise, PIPs form primary channels mediating efficient water uptake and thereby control plasma membrane potentials of permeability, while TIPs, in addition to their high water transport capacity in tonoplasts [60], also transport CO2 [13] and urea [12].

In the present work, SIP and NIP subfamilies were less responsive to the applied stress. We noticed, that NIPs were not up-regulated in the sensitive bulks $\mathrm{t}$ SD24S versus SDSC (Figure 3(b)), as well as among the down-regulated unitags 
in the tolerant bulks SD24T versus SD24S (Figure 3(c)). On the other hand, the SIP subfamily also harbored no isoform among the down-regulated unitags in the contrast SD24T versus SDTC (Figure 3(a)). Alexandersson et al. [51] also confirmed that some SIP isoforms presented little expression variation in Arabidopsis plants under drought stress (watering suppression extended until 12 days). Therefore, AtSIP1-1 was considered as constitutively expressed. This low responsiveness to water deficit can be explained by the unique location of these aquaporins in the endoplasmic reticulum [59], an organelle with tortuous structure and high surface-to-volume ratio with high demand for osmotic balance volume and, therefore, may not require the water transport mediated by aquaporins [59]. Thus, further studies are necessary to define SIP functions more clearly.

The level of aquaporin transcripts varied less than 10 times based on the unitags in the contrasts, except for the SD173276 unitag (a potential SoTIP2-2), which was almost two thousand times higher in the tolerant SDTC versus SDSC contrast, and almost 500 times in the sensitive contrast SD24S versus SDSC (data not shown).

Alexandersson et al. [51] also observed that most aquaporins do not alter their expression under water deficit stress, and no Arabidopsis aquaporin isoforms varied their expression more than twice until the seventh day of stress treatment.

The contrast analysis of the tolerant bulks defined four possible targets: one exclusively up-regulated PIP1-1 isoform (SD264077 unitag, FC 3.58), and three exclusively down-regulated PIP2-2s (SD176950 unitag, FC -2.34), PIP21 (SD176664 unitag, FC -1.73) and NIP1-1 (SD202395 unitag, FC -1.36).

In rice, the PIP1-1 isoform promoted salt stress tolerance [23], and it was involved in the rehydration after cooling stress in tolerant genotypes [61]. PIP1-1 overexpression conferred tolerance to water deficit in rice and to salt stress in transgenic Arabidopsis [62]. This isoform also responded to drought and daytime in grapevine [56]. The up-regulated SD264077 unitag, as a possible PIP1-1 isoform, was validated in the present work by RT-qPCR analysis, as detailed in the next chapter, and represents a potential target for further studies, including the development of molecular markers for marker-assisted selection in breeding (real-time PCRassisted selection) [63] or cis-genesis (insertion of genes in different accessions of the same species [64]), already successfully applied by Joshi et al. [65]. These authors inserted resistance genes to apple scab under the control of the RubisCO promoter in varieties known to be susceptible to the pathogen.

Isoform PIP2-2 is down-regulated over four times in Arabidopsis under 12 days of drought [51] and in barley under salt stress [66]. As expected, it was also observed in the tolerant bulk analysis of the present study, showing FC -2.34. The subsequent PIP2-2 monitoring revealed that aquaporin expression increased sensitivity to salt stress in transgenic rice [67]. This point is relevant, since crosstalks involving shared pathways in response to drought and salinity stress are regular $[68,69]$. Thus, this isoform, after appropriate
RT-qPCR validation, could be useful as stress-indicator in breeding programs.

On the other hand, the potential usefulness of unitags related to PIP2-1, PIP2-5, PIP2-6, TIP1-1, TIP2-2, SIP1-1, and SIP1-2 in breeding programs still need to be confirmed. In relation to the PIP2-5 isoform (up- and down-regulated simultaneously in the present study, depending on unitags), Jang et al. [70] observed that overexpressing this aquaporin reduced drought tolerance of transgenic Arabidopsis and tobacco. The same group proposed that PIP2-5 expression influenced the transcription levels of other PIPs and $\mathrm{H}+-$ ATPases (enzymes that regulate the cytoplasmic $\mathrm{pH}$ in which levels of $\mathrm{H}+$ interfere with the control of the opening and closing of the aquaporins channels known as the aquaporin gating [71]). Lembke et al. [25] also observed this isoform to be down-regulated under water deficit (72 hours of watering suppression), despite the detected induction via oligonucleotide array hybridization.

Therefore, for the tolerant bulk of genotypes, this isoform is expected to restrain its expression under root dehydration (24 h).

Basically, up- or down-regulation and constitutive expression were all observed in the contrast analysis of tolerant bulks, (except that down-regulation was not observed in the SIP subfamily; Figure 3(a)).

The sensitive bulk of genotypes also presented all three expression levels for each aquaporin subfamily (with the exception of the NIP subfamily, in which up-regulation was not observed; Figure 3(b)). The analysis of the sensitive bulks allowed the identification of only up-regulated [PIP22 (SD176665 unitag, FC 4.83), PIP2-4 (SD176663 unitag, FC 1.66), PIP2-6 (SD176669 unitag, FC 1.70)] or only down-regulated [PIP1-1 (SD264077 unitag, FC -4.56), NIP11 (SD202395 unitag, FC -2.00)] aquaporin isoforms. These exclusively up- or down-regulated isoforms, respectively, may represent a panel of markers based on real-time PCR, and suggesting high stress sensitivity. In this way, at least two candidates are particularly appealing: (a) PIP1-1, that was upregulated (SD264077 unitag, FC 3.58) in the tolerant bulks and entirely differently regulated (SD264077 unitag, FC 4.56) in the sensitive bulks; (b) PIP2-2 isoform, which was exclusively down-regulated in the tolerant bulks (SD176950 unitag, FC -2.34) and up-regulated in the sensitive bulks (SD176665 unitag, FC 4.83). Thus, both isoforms are strong candidates for further research aiming at molecular marker development and cis-genesis. Finally, further studies are needed to determine the true meaning of each stressresponsive isoform.

When comparing both genotype bulks under stress (SD24T versus SD24S), all three expression levels (up- or down-regulation and constitutive expression) were observed for each aquaporin isoform subfamily. Notably, a specific isoform in the SIP subfamily was down-regulated in the tolerant bulk, but not in the sensitive bulk (Figure 3(c)). Two more isoforms are worth mentioning: PIP2-4 (SD176664 unitag, FC 3.64), which was more transcribed in the stressed tolerant bulk than in the stressed sensitive one, and PIP2-1 (SD176669 unitag, FC -1.13), which is being less transcribed 
TABLE 4: Aquaporin unitags with distinct expression rates in root HT-SuperSAGElibraries from contrasts of tolerant ${ }^{\mathrm{T}}$ (SD24T versus SDTC) and sensitive ${ }^{S}$ (SD24S versus SDSC) sugarcane genotypes and after continuous dehydration $(24 \mathrm{~h})$.

\begin{tabular}{|c|c|c|c|c|c|}
\hline Unitag & Anotation & $\mathrm{FC}^{\mathrm{T}}$ & $\begin{array}{l}\text { Unitag } \\
\text { regulation }\end{array}$ & $\mathrm{FC}^{\mathrm{S}}$ & $\begin{array}{l}\text { Unitag } \\
\text { regulation }\end{array}$ \\
\hline SD264077 & SsPIP1-1 & 3.58 & UR & -4.56 & $\mathrm{DR}$ \\
\hline SD2444 & SoPIP1-3/PIP1-4 & 5.93 & UR & -2.66 & $\mathrm{DR}$ \\
\hline SD231548 & SoPIP1-3/PIP1-4 & 3.18 & UR & 1.28 & n.s. \\
\hline SD243866 & SoPIP2-1 & 13.51 & UR & -15.97 & $\mathrm{DR}$ \\
\hline SD243874 & SoPIP2-1 & 3.18 & UR & -2.14 & $\mathrm{DR}$ \\
\hline SD28082 & SoPIP2-4 & 15.10 & UR & -6.39 & $\mathrm{DR}$ \\
\hline SD28080 & SoPIP2-4 & 1.23 & UR & -1.39 & n.s. \\
\hline SD198883 & SoPIP2-5 & 1.46 & UR & -1.25 & n.s. \\
\hline SD36536 & SoTIP1-1 & 2.97 & UR & -1.90 & n.s. \\
\hline SD80612 & SoTIP1-1 & 1.11 & UR & -1.07 & n.s. \\
\hline SD84958 & SsTIP1-1 & 2.02 & UR & -1.82 & $\mathrm{DR}$ \\
\hline SD36243 & $H v$ TIP2-2 & 3.53 & UR & 1.34 & n.s. \\
\hline SD182891 & SoTIP2-2 & 2.78 & UR & -5.85 & $\mathrm{DR}$ \\
\hline SD119963 & SoTIP2-3 & 5.56 & UR & 1.93 & n.s. \\
\hline SD119859 & SoTIP2-3 & 3.18 & UR & -2.66 & $\mathrm{DR}$ \\
\hline SD217703 & SoNIP3-1 & 1.93 & UR & -1.01 & n.s. \\
\hline SD21811 & SoSIP1-1 & 1.65 & UR & 1.01 & n.s. \\
\hline SD96919 & SoSIP1-2 & 2.38 & UR & -1.06 & n.s. \\
\hline SD233575 & SoPIP1-3/PIP1-4 & -6.56 & $\mathrm{DR}$ & 4.21 & UR \\
\hline SD231438 & SoPIP1-3/PIP1-4 & -5.16 & $\mathrm{DR}$ & 1.38 & n.s. \\
\hline SD231437 & SoPIP1-3/PIP1-4 & -1.89 & $\mathrm{DR}$ & 1.15 & UR \\
\hline SD205705 & SoPIP1-3/PIP1-4 & -1.87 & $\mathrm{DR}$ & -1.14 & n.s. \\
\hline SD231440 & SoPIP1-3/PIP1-4 & -2.79 & $\mathrm{DR}$ & -2.14 & n.s. \\
\hline SD241279 & SoPIP1-5 & -1.22 & $\mathrm{DR}$ & 2.40 & UR \\
\hline SD91837 & SoPIP2-4 & -17.81 & $\mathrm{DR}$ & 1.32 & n.s. \\
\hline SD243847 & SoPIP2-1 & -7.50 & $\mathrm{DR}$ & -1.60 & n.s. \\
\hline SD243911 & SoPIP2-1 & -5.62 & DR & -1.33 & n.s. \\
\hline SD54851 & SoPIP2-1 & -2.53 & DR & 1.87 & n.s. \\
\hline SD176663 & OsPIP2-4 & -2.51 & $\mathrm{DR}$ & 1.66 & UR \\
\hline SD176664 & SoPIP2-4 & -1.73 & $\mathrm{DR}$ & 1.02 & n.s. \\
\hline SD84616 & SoPIP2-5 & -4.42 & $\mathrm{DR}$ & 1.32 & n.s. \\
\hline SD176669 & SoPIP2-6 & -2.15 & $\mathrm{DR}$ & 1.70 & UR \\
\hline SD19005 & $H v$ TIP1-1 & -1.87 & $\mathrm{DR}$ & 1.84 & n.s. \\
\hline SD19006 & $H v$ TIP1-1 & -5.16 & $\mathrm{DR}$ & 4.67 & UR \\
\hline SD7939 & TaTIP1-1 & -2.95 & $\mathrm{DR}$ & 1.76 & n.s. \\
\hline SD80616 & SsTIP1-1 & -2.81 & $\mathrm{DR}$ & 2.34 & UR \\
\hline SD182871 & SoTIP2-2 & -6.09 & $\mathrm{DR}$ & -1.63 & n.s. \\
\hline SD173276 & SoTIP2-2 & -3.33 & DR & 496.29 & UR \\
\hline SD119919 & SoTIP2-3 & -6.56 & DR & 1.61 & n.s. \\
\hline SD194892 & SoNIP3-1 & -2.34 & $\mathrm{DR}$ & -1.60 & n.s. \\
\hline
\end{tabular}

Isoforms are preceded by the abbreviated species name (Hv: Hordeum vulgare; Os: Oryza sativa; So: Saccharum officinarum; Ss: Saccharum spp. and Ta: Triticum aestivum). HT-SuperSAGE libraries: SD24T (bulk of tolerant genotypes under stress; SD24S (bulk of sensitive genotypes under stress); SDTC (control bulk of tolerant genotypes); and SDSC (control bulk of sensitive genotypes). FC: ratio of the frequencies (normalized to 1,000,000) observed in the stressed library in relation to the control library. DR: down-regulated; UR: up-regulated; n.s.: not significant at $P<0.05$. 


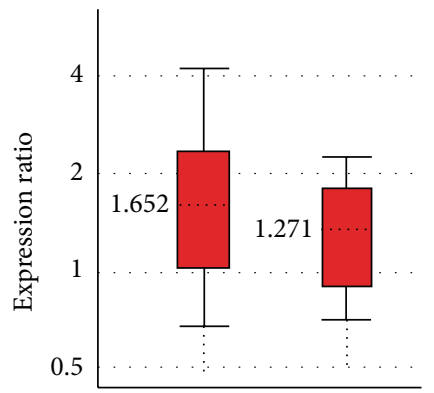

(a)

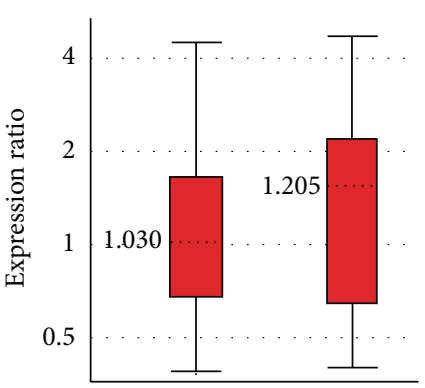

(e)

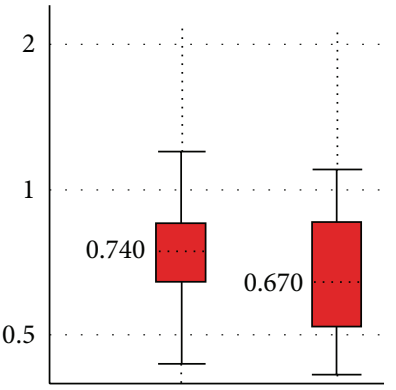

(b)

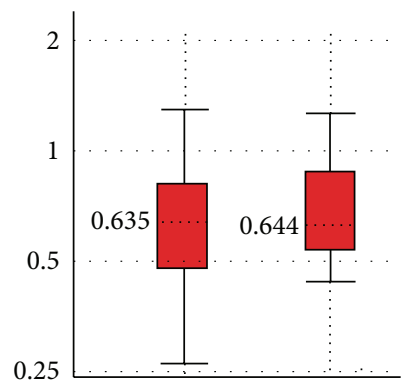

(f)

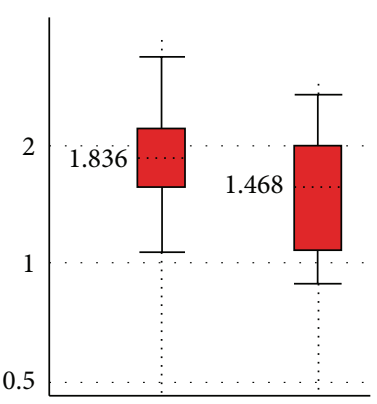

(c)

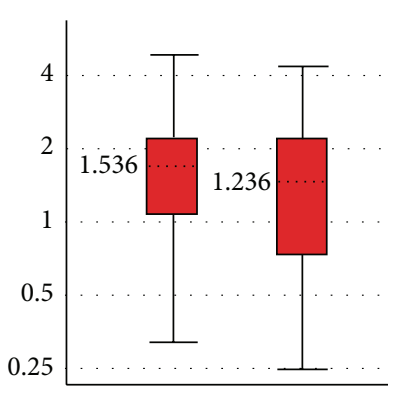

(g)

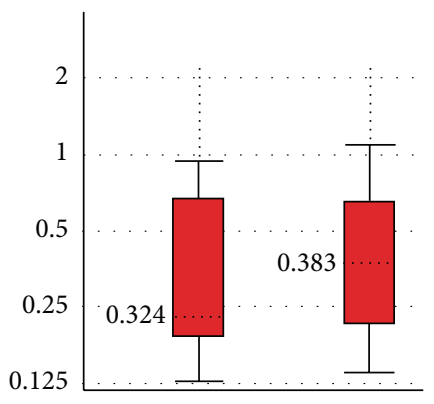

(d)

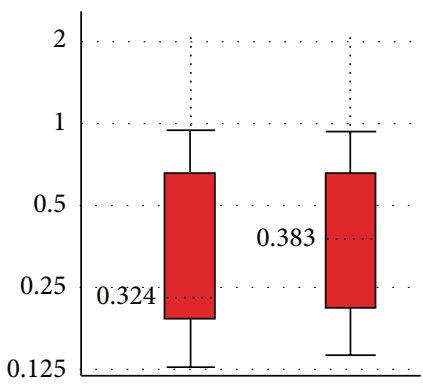

(h)

FIGURE 4: Relative quantification of SsPIP1 aquaporin-1 (unitag SD264077) and SoPIP1-3/PIP1-4 (unitag SD231548) with sugarcane cDNAs [tolerant genotypes: (a) CTC6, (b) CTC15, (c) SP83-2847, (d) SP83-5073; sensitive genotypes [(e) CTC9, (f) CTC13, (g) SP90-1638, (h) SP903414] under stress ( $24 \mathrm{~h}$ of continuous dehydration). The expression ratios of the same genotypes under control condition were normalized by reference genes GAPDH and $25 \mathrm{~S}$ rRNA. ${ }^{*}$ Relative expression (REST ${ }^{\odot}$ v. 2.0 .13 software) with the mean value (horizontally dotted line) and range of the observations (100\%, horizontal bars); in red: confidence interval at $95 \%$.

in the stressed tolerant bulk as compared to the stressed sensitive bulk.

The unitag related to PIP2-4 (SD176664) was downregulated in the tolerant bulks after onset of the stress, and it had no relevant expression changes in the sensitive bulks. Nevertheless, it was more expressed in the tolerant bulk when compared with the sensitive bulk, upon stress or even under control conditions. Thus, the tolerant genotypes seemed to produce more PIP2-4 transcripts than the sensitive genotypes. In maize, this aquaporin isoform was up-regulated after only two hours of salt stress, in which time the recovery phase of the osmotic potential falls [52].

In turn, the PIP2-1-related unitag (SD176669) behaved differently under stress and its reaction depends on the genotype (it was down-regulated as compared to the tolerant bulks, and up-regulated as compared to the sensitive bulks). When considering the contrast between both control and stressed bulks of genotypes, this unitag was better expressed in the sensitive bulk than in the tolerant one. By taking into account that this aquaporin isoform increases insensitivity to salinity [67] in transgenic rice and the fact that salinity and drought share many response pathways $[68,69]$, this isoform deserves further investigation.

Analysis of controls of the two different genotype bulks $s$ showed that the aquaporin isoforms are present in all subfamilies and expressed in the three studied levels during normal daily irrigation (Figure 3(d)). Four isoforms [PIP1-2 (SD92576 unitag); PIP2-1 (SD176664 unitag);
PIP2-4 (SD176663 and SD176950 unitags); PIP2-6 (SD176669 unitag)] presented significantly higher abundance in the tolerant bulk, while only one (NIP1-1) was less expressed in relation to the sensitive bulk. In turn, five isoforms (PIP2-2, PIP2-3, PIP2-5, PIP2-6, and TIP4-2) were similarly transcribed in both bulks, while another five isoforms (PIP21, PIP2-4, TIP2-2, TIP2-3, and SIP1-2) presented all the three expression levels. Differences in the transcriptional profiles of both controls bulks reinforce the expression modulation of genes presenting in the genotypes composing the bulks.

The two main comparisons SD24T versus SDTC and SD24S versus SDSC revealed that from a total of 18 upregulated unitags in the tolerant bulks, eight were downregulated in sensitive bulks, while from 22 other unitags, down-regulated in the tolerant bulks, eight were up-regulated in the sensitive bulks (Table 4). The same isoforms showing different expression levels (Table 4) can be explained by the similarities between aquaporins sequences, in part a consequence of the high level of duplicated plant MIP genes, which is higher than that observed in vertebrates, possibly reflecting the environmental pressures plants are exposed to $[45,49]$, and also the aneuploidy and polyploidy events observed in the Saccharum complex [41].

3.4. Unitag Expression Validation by RT-qPCR. The use of RT-qPCR for the confirmation of aquaporin gene expression changes in grass (maize [72] and sugarcane [25]) has already been reported. In the present work we attempted to 
TABLE 5: Primers sequences for RT-qPCR of SsPIP1-1, SoPIP1-3/PIP1-4 (designed from sugarcane ESTs), 25S rRNA, and GAPDH (as reference genes).

\begin{tabular}{|c|c|c|c|c|c|c|c|c|}
\hline Unitag & EST/cluster & Database* & Gene & Primers & $\begin{array}{l}\mathrm{Tm} \\
\left({ }^{\circ} \mathrm{C}\right)\end{array}$ & $\begin{array}{l}\text { Amplicon } \\
\quad(\mathrm{bp})\end{array}$ & $\begin{array}{c}\text { Regression } \\
\text { coefficient } \\
\left(R^{2}\right)\end{array}$ & $\begin{array}{l}\text { Amplification } \\
\text { efficiency }(\%)\end{array}$ \\
\hline SD264077 & gi|35203438 & $\mathrm{dbEST}$ & SsPIP1-1 & $\begin{array}{l}5^{\prime} \text {-GTTCCTATCCTTGCCCCACT-3' } \\
3^{\prime} \text {-AGGCGTGATCCCTGTTGTAG-5' }\end{array}$ & 84.6 & 134 & 0.995 & 95.55 \\
\hline SD231548 & TC127588 & SoGI & $\begin{array}{l}\text { SoPIP1- } \\
\text { 3/PIP1-4 }\end{array}$ & $\begin{array}{l}5^{\prime} \text {-GACTCCCATGTTCCTATCCTTG-3' } \\
3^{\prime} \text {-CGTGATCCCTGTTGTAGATGAT-5' }\end{array}$ & 84.3 & 142 & 0.992 & 93.47 \\
\hline- & gi|33464288 & dbEST & $\begin{array}{c}25 \mathrm{~S} \\
\text { rRNA }\end{array}$ & $\begin{array}{l}5^{\prime} \text {-GCAGCCAAGCGTTCATAG-3' } \\
3^{\prime} \text { - CGGCACGGTCATCAGTAG-5' }\end{array}$ & 82.9 & 172 & 0.999 & 99.82 \\
\hline - & TC531505 & SoGI & GAPDH & $\begin{array}{l}5^{\prime} \text {-GGTTCACTTGAAGGGTGGTG-3' } \\
3^{\prime} \text { - TGAGGTGTACCTGTCCTCGTT-5' }\end{array}$ & 81.8 & 100 & 0.984 & 100.89 \\
\hline
\end{tabular}

Isoforms are preceded by the abbreviated species name (So: Saccharum officinarum; Ss: Saccharum spp.).

${ }^{*}$ Databases: dbEST (NCBI; http://www.ncbi.nlm.nih.gov/), Gene Index (SoGI; http://compbio.dfci.harvard.edu/tgi/)].

determine which genotype was responsible for the bulk of expression in tolerant or sensitive genotypes. To that end, each genotype composing that bulk was independently tested by RT-qPCR analysis. Thus, two unitags [SD264077 (PIP1-1) and SD231548 (PIP1-3/PIP1-4)] considered UR in the tolerant bulk as well as DR and n.s. in the sensitive were selected for expression validation using two reference genes (25S rRNA and GAPDH), both reported to be suitable for sugarcane (Table 5). The relative expression results of the tolerant and sensitive genotypes for the two target genes are shown in Table 6, together with their respective unitag results.

PIP1-1 (SD264077 unitag) was induced by stress in two of the tolerant genotypes (CTC6 and SP83-2847), in comparison to the respective controls (Table 6, Figure 4). Nevertheless, in the remaining tolerant genotypes (CTC15 and SP83-5073) both PIP1-1 genes were down-regulated under the same conditions (Table 6, Figure 4). Thus it can be concluded that CTC6 and SP83-2847 were responsible for the unitag upregulation. The overexpression of rice PIP1-1 in root and leaf (within $24 \mathrm{~h}$ ) enhanced the tolerance to drought $(200 \mathrm{mM}$ mannitol) and salt stress (100 $\mathrm{mM} \mathrm{NaCl})$ in transgenic Arabidopsis [62]. Also, PIP1-1 aquaporin isoforms in grapevine were highly expressed in roots (RT-qPCR) in response to water deficit (8 days of constant dehydration [56]).

PIP1-3/PIP1-4 (SD231548 unitag), were stress-induced in genotype SP83-2847 (Table 6, Figure 4), in agreement with the HT-SuperSAGE data. It should be noted that in phylogenetic analyses PIP1-3 and PIP1-4 are highly similar with barley PIP amino acid sequences, being grouped together as one isoform, while they are phylogenetically more distant from PIP1-1 (from barley and rice, [73]). A. thaliana PIP1-3 and PIP1-4 isoforms had their transcription level increased more than five times, covering the first $48 \mathrm{~h}$ of drought stress ( $250 \mathrm{mM}$ mannitol), in leaves and roots, as well as in response to salt $(150 \mathrm{mM} \mathrm{NaCl})$ and cold stresses [48]. In turn, PIP13 overexpression in transgenic rice, combining aquaporin coding sequence with a constitutive corn promoter, showed enhanced stress tolerance to cold [74].

However, in relation to water transport by PIP1-3, which appears to be less permeable to water [74], this isoform could work best in conjunction with PIP2 subgroup members, in
TABLE 6: Relative expression rates of aquaporins PIP1-1 (SD264077 unitag) and PIP1-3/PIP1-4 (SD231548 unitag) in bulks of tolerant or sensitive genotypes, respectively, and RT-qPCR data (both in bulks and each genotype).

\begin{tabular}{|c|c|c|}
\hline \multirow{3}{*}{ Technique and genotypes } & \multicolumn{2}{|c|}{ Target gene } \\
\hline & PIP1-1 & PIP1-3/PIP1-4 \\
\hline & \multicolumn{2}{|c|}{ Modulation of gene expression } \\
\hline \multicolumn{3}{|l|}{ HT-SuperSAGE } \\
\hline Tolerant bulk ${ }^{1}$ & $3.580^{*} / \mathrm{UR}$ & $3.180^{*} / \mathrm{UR}$ \\
\hline Sensitive bulk ${ }^{1}$ & $-4.560^{*} / \mathrm{DR}$ & $1.280^{*} / \mathrm{ns}$ \\
\hline \multicolumn{3}{|l|}{$\begin{array}{l}\text { RT-qPCR and Tolerant } \\
\text { Genotypes }\end{array}$} \\
\hline CTC6 & $1.652^{\#} / \mathrm{UR}$ & $1.271^{\#} / \mathrm{ns}$ \\
\hline CTC15 & $0.740^{\#} / \mathrm{DR}$ & $0.670^{\#} / \mathrm{DR}$ \\
\hline SP83-2847 & $1.836^{\# / \mathrm{UR}}$ & $1.468^{\# / \mathrm{UR}}$ \\
\hline SP83-5073 & $0.324^{\#} / \mathrm{DR}$ & $0.383^{\#} / \mathrm{DR}$ \\
\hline \multicolumn{3}{|l|}{$\begin{array}{l}\text { RT-qPCR and sensitive } \\
\text { Genotypes }\end{array}$} \\
\hline СТС9 & $1.030^{\# / \mathrm{ns}}$ & $1.205^{\# / \mathrm{ns}}$ \\
\hline CTC13 & $0.635^{\#} / \mathrm{DR}$ & $0.644^{\#} / \mathrm{DR}$ \\
\hline SP90-1638 & $1.536^{\#} / \mathrm{ns}$ & $1.236^{\# / \mathrm{ns}}$ \\
\hline SP90-3414 & $0.324^{\#} / \mathrm{DR}$ & $0.383^{\#} / \mathrm{DR}$ \\
\hline
\end{tabular}

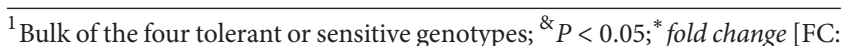
ratio of the frequencies (normalized to 1,000,000) observed in the stressed library in relation to the control library]; ${ }^{\#}$ relative expression level using the REST software (v. 2.0.13) [26]; DR: down-regulated; UR: up-regulated; ns: not significant at $P<0.05$.

which in silico analysis showed them to be mostly DR. Considering the remaining components of the tolerant bulk, the genotype CTC6 did not show significant differences in transcript levels, while CTC15 and SP83-5073 presented down-regulated transcription at the onset of root dehydration (Table 6, Figure 4).

The strategy of opening bulks in the RT-qPCR validation reinforced the transcription modulation of sugarcane aquaporins and gave hints to genotype-specific expression. Thus, plants considered physiologically tolerant or sensitive to root 
dehydration $(24 \mathrm{~h})$ varied in the expression of aquaporin isoforms. The same was observed with up (O. sativa L. cv. Zhonghan 3) and lowland (cv. Xiushui) rice under water deficit [75]. The RT-qPCR results revealed genotype-specific differences for PIP1-2, PIP1-3, PIP2-1, and PIP2-5 isoforms in roots, and PIP1-2 and PIP1-3 in leaves. The above mentioned isoforms were up-regulated in upland rice, whereas they remained unchanged or DR in lowland rice [75].

Finally, the RT-qPCR protocol, in the present work applied for unitags validation, as well as the identified unitags for PIP1-1 and PIP 1-3/PIP1-4, define a set of functional molecular markers based on the expression profiles validated with appropriate genotypes. This expression marker set will assist breeders in marker-assisted selection of elite genotypes more tolerant to abiotic stresses.

\section{Conclusions}

The present work is a pioneer study specifically addressing the aquaporin transcripts in sugarcane transcriptomes established from HT-SuperSAGE transcription profiles from roots of tolerant and sensitive genotypes after $24 \mathrm{~h}$ of continuous dehydration. Almost all 26 bp unitags were annotated using a public sugarcane EST databases, especially S. by $S$., allowing the identification of potential aquaporins. Categorizing the EST-anchored unitags by Gene Ontology (GO) enhanced the annotation efficiency by almost $15 \%$. These procedures identified potential isoforms of the four aquaporin subfamilies (PIP, TIP, NIP, and SIP) already described for higher plants, together with their respective expression profiles in sugarcane under abiotic stress. Moreover, an efficient protocol for RTqPCR was developed, enabling gene expression validation of SuperSAGE unitags related to PIP aquaporins (PIP1-1 and PIP1-3/PIP1-4) and involving reference genes encoding GAPDH and 25S rRNA, testing each genotype individually the employed, validation strategy revealed genotypespecificity of the response to the applied stress.

\section{Conflict of Interests}

The authors declare that there is no conflict of interests.

\section{Acknowledgments}

The authors thank Dr. Björn Rotter, Ruth Jungmann, Nicolas Krezdorn, and Dr. Peter Winter (GenXPro GmbH, Germany) for scientific and technical advices. This work has been funded by Brazilian institutions Financiadora de Estudos e Projetos (FINEP), Fundação de Amparo à Ciência e Tecnologia do Estado de Pernambuco (FACEPE), and Conselho Nacional de Desenvolvimento Científico e Tecnológico (CNPq).

\section{References}

[1] G. E. Welbaum and F. C. Meinzer, "Compartmentation of solutes and water in developing sugarcane stalk tissue," Plant Physiology, vol. 93, no. 3, pp. 1147-1153, 1990.
[2] S. L. Dillon, F. M. Shapter, R. J. Henry, G. Cordeiro, L. Izquierdo, and L. S. Lee, "Domestication to crop improvement: genetic resources for Sorghum and Saccharum (Andropogoneae)," Annals of Botany, vol. 100, no. 5, pp. 975-989, 2007.

[3] FAOSTAT, "Food and Agriculture Organization of the Unite Nations. In: FAO statistical databases," 2011, http:// faostat.fao.org/.

[4] S. S. Gosal, S. H. Wani, and M. S. Kang, "Biotechnology and drought tolerance," Journal of Crop Improvement, vol. 23, no. 1, pp. 19-54, 2009.

[5] M. D. A. Silva, J. A. G. Da Silva, J. Enciso, V. Sharma, and J. Jifon, "Yield components as indicators of drought tolerance of sugarcane," Scientia Agricola, vol. 65, no. 6, pp. 620-627, 2008.

[6] E. A. Bray, "Molecular responses to water deficit," Plant Physiology, vol. 103, no. 4, pp. 1035-1040, 1993.

[7] K. Shinozaki and K. Yamaguchi-Shinozaki, "Gene networks involved in drought stress response and tolerance," Journal of Experimental Botany, vol. 58, no. 2, pp. 221-227, 2007.

[8] S. D. Tyerman, C. M. Niemietz, and H. Bramley, "Plant aquaporins: multifunctional water and solute channels with expanding roles," Plant, Cell and Environment, vol. 25, no. 2, pp. 173-194, 2002.

[9] C. Maurel, "Plant aquaporins: novel functions and regulation properties," FEBS Letters, vol. 581, no. 12, pp. 2227-2236, 2007.

[10] M. Besse, T. Knipfer, A. J. Miller, J.-L. Verdeil, T. P. Jahn, and W. Fricke, "Developmental pattern of aquaporin expression in barley (Hordeum vulgare L.) leaves," Journal of Experimental Botany, vol. 62, no. 12, pp. 4127-4142, 2011.

[11] P. Gerbeau, J. Güçlü, P. Ripoche, and C. Maurel, "Aquaporin Nt-TIPa can account for the high permeability of tobacco cell vacuolar membrane to small neutral solutes," Plant Journal, vol. 18 , no. 6 , pp. $577-587,1999$.

[12] L.-H. Liu, U. Ludewig, B. Gassert, W. B. Frommer, and N. Von Wirén, "Urea transport by nitrogen-regulated tonoplast intrinsic proteins in arabidopsis," Plant Physiology, vol. 133, no. 3, pp. 1220-1228, 2003.

[13] N. Uehleln, C. Lovisolo, F. Siefritz, and R. Kaldenhoff, "The tobacco aquaporin NtAQP1 is a membrane $\mathrm{CO}_{2}$ pore with physiological functions," Nature, vol. 425, no. 6959, pp. 734-737, 2003.

[14] D. Loqué, U. Ludewig, L. Yuan, and N. Von Wirén, “Tonoplast intrinsic proteins AtTIP2;1 and AtTIP2;3 facilitate $\mathrm{NH}_{3}$ transport into the vacuole," Plant Physiology, vol. 137, no. 2, pp. 671680, 2005.

[15] C. Dordas, M. J. Chrispeels, and P. H. Brown, "Permeability and channel-mediated transport of boric acid across membrane vesicles isolated from squash roots," Plant Physiology, vol. 124, no. 3, pp. 1349-1361, 2000.

[16] T. Henzler and E. Steudle, "Transport and metabolic degradation of hydrogen peroxide in chara corallina: model calculations and measurements with the pressure probe suggest transport of $\mathrm{H}_{2} \mathrm{O}_{2}$ across water channels," Journal of Experimental Botany, vol. 51, no. 353, pp. 2053-2066, 2000.

[17] G. P. Bienert, M. Thorsen, M. D. Schüssler et al., "A subgroup of plant aquaporins facilitate the bi-directional diffusion of $\mathrm{As}(\mathrm{OH})_{3}$ and $\mathrm{Sb}(\mathrm{OH})_{3}$ across membranes," BMC Biology, vol. 6, article 26, 2008.

[18] U. Johanson and S. Gustavsson, "A new subfamily of major intrinsic proteins in plants," Molecular Biology and Evolution, vol. 19, no. 4, pp. 456-461, 2002. 
[19] M. S. Lucero, F. Mirarchi, J. Goldstein, and C. Silberstein, "Intraperitoneal administration of Shiga toxin 2 induced neuronal alterations and reduced the expression levels of aquaporin 1 and aquaporin 4 in rat brain," Microbial Pathogenesis, vol. 53, no. 2, pp. 87-94, 2012.

[20] G. Di Giusto, P. Flamenco, V. Rivarola et al., "quaporin 2increased renal cell proliferation is associated with cell volume regulation," Journal of Cellular Biochemistry, vol. 113, no. 12, pp. 3721-3729, 2012.

[21] C. Geijer, D. Ahmadpour, M. Palmgren et al., "Yeast aquaglyceroporins use the transmembrane core to restrict glycerol transport," The Journal of Biological Chemistry, vol. 287, no. 28, pp. 23562-23570, 2012.

[22] M. Ayadi, D. Cavez, N. Miled, F. Chaumont, and K. Masmoudi, "Identification and characterization of two plasma membrane aquaporins in durum wheat (Triticum turgidum L. subsp. durum) and their role in abiotic stress tolerance," Plant Physiology and Biochemistry, vol. 49, no. 9, pp. 1029-1039, 2011.

[23] C. Liu, T. Fukumoto, T. Matsumoto et al., "Aquaporin OsPIP1;1 promotes rice salt resistance and seed germination," Plant Physiology and Biochemistry, vol. 63, pp. 151-158, 2013.

[24] C. Maure, L. Verdoucq, D.-T. Luu, and V. Santoni, "Plant aquaporins: membrane channels with multiple integrated functions," Annual Review of Plant Biology, vol. 59, pp. 595-624, 2008.

[25] C. G. Lembke, M. Y. Nishiyama Jr., P. M. Sato, R. F. Andrade, and G. M. Souza, "Identification of sense and antisense transcripts regulated by drought in sugarcane," Plant Molecular Biology, vol. 79, no. 4-5, pp. 461-477, 2012.

[26] M. W. Pfaffl, G. W. Horgan, and L. Dempfle, "Relative expression software tool (REST) for group-wise comparison and statistical analysis of relative expression results in real-time PCR," Nucleic Acids Research, vol. 30, no. 9, p. e36, 2002.

[27] K. Tamura, D. Peterson, N. Peterson, G. Stecher, M. Nei, and S. Kumar, "MEGA5: molecular evolutionary genetics analysis using maximum likelihood, evolutionary distance, and maximum parsimony methods," Molecular Biology and Evolution, vol. 28, no. 10, pp. 2731-2739, 2011.

[28] H. Matsumura, D. H. Krüger, G. Kahl, and R. Terauchi, "SuperSAGE: a modern platform for genome-wide quantitative transcript profiling," Current Pharmaceutical Biotechnology, vol. 9, no. 5, pp. 368-374, 2008.

[29] H. Matsumura, S. Reich, A. Ito et al., "Gene expression analysis of plant host-pathogen interactions by SuperSAGE," Proceedings of the National Academy of Sciences of the United States of America, vol. 100, no. 26, pp. 15718-15723, 2003.

[30] H. Matsumura, S. Reich, M. Reuter et al., "perSAGE: a potent transcriptome tool for eukaryotic organisms," in Current Technologies and Applications, S. M. Wang, Ed., pp. 77-90, Horizon Scientific Press, Norwich, UK, 2004.

[31] H. Matsumura, K. Yoshida, S. Luo et al., "High-throughput SuperSAGE," Methods in Molecular Biology, vol. 687, pp. 135146, 2011.

[32] H. Matsumura, N. Urasaki, K. Yoshida, D. H. Kruger, G. Kahl, and R. Terauchi, "SuperSAGE: powerful serial analysis of gene expression," Methods in Molecular Biology, vol. 883, pp. 1-17, 2012.

[33] E. A. Kido, J. R. C. Ferreira Neto, R. L. O. Silva et al., "New insights in the sugarcane transcriptome responding to drought stress as revealed by SuperSAGE," The Scientific World Journal, vol. 2012, pp. 1-14, 2012.
[34] S. Audic and J.-M. Claverie, "The significance of digital gene expression profiles," Genome Research, vol. 7, no. 10, pp. 986995, 1997.

[35] N. Robertson, M. Oveisi-Fordorei, S. D. Zuyderduyn et al., "DiscoverySpace: an interactive data analysis application," Genome Biology, vol. 8, no. 1, article R6, 2007.

[36] A. Conesa, S. Götz, J. M. García-Gómez, J. Terol, M. Talón, and M. Robles, "Blast2GO: a universal tool for annotation, visualization and analysis in functional genomics research," Bioinformatics, vol. 21, no. 18, pp. 3674-3676, 2005.

[37] J. D. Thompson, D. G. Higgins, and T. J. Gibson, "CLUSTAL W: improving the sensitivity of progressive multiple sequence alignment through sequence weighting, position-specific gap penalties and weight matrix choice," Nucleic Acids Research, vol. 22, no. 22, pp. 4673-4680, 1994.

[38] S. Rozen and H. Skaletsky, "Primer3 on the WWW for general users and for biologist programmers," Methods in Molecular Biology, vol. 132, pp. 365-386, 2000.

[39] E. A. Kido, P. K. de Araújo Barbosa, J. R. C. F. Neto et al., "Identification of plant protein kinases in response to abiotic and biotic stresses using superSAGE," Current Protein and Peptide Science, vol. 12, no. 7, pp. 643-656, 2011.

[40] A. D’Hont, L. Grivet, P. Feldmann, S. Rao, N. Berding, and J. C. Glaszmann, "Characterisation of the double genome structure of modern sugarcane cultivars (Saccharum spp.) by molecular cytogenetics," Molecular and General Genetics, vol. 250, no. 4, pp. 405-413, 1996.

[41] L. Grivet and P. Arruda, "Sugarcane genomics: depicting the complex genome of an important tropical crop," Current Opinion in Plant Biology, vol. 5, no. 2, pp. 122-127, 2002.

[42] A. L. Vettore, F. R. Da Silva, E. L. Kemper, and P. Arruda, "The libraries that made SUCEST," Genetics and Molecular Biology, vol. 24, no. 1-4, pp. 1-7, 2001.

[43] F. Chaumont, F. Barrieu, E. Wojcik, M. J. Chrispeels, and R. Jung, "Aquaporins constitute a large and highly divergent protein family in maize," Plant Physiology, vol. 125, no. 3, pp. 1206-1215, 2001.

[44] M. X. Nguyen, S. Moon, and K. H. Jung, "Genome-wide expression analysis of rice aquaporin genes and development of a functional gene network mediated by aquaporin expression in roots," Planta, vol. 238, no. 4, pp. 669-681, 2013.

[45] G. Soto, K. Alleva, G. Amodeo, J. Muschietti, and N. D. Ayub, "New insight into the evolution of aquaporins from flowering plants and vertebrates: orthologous identification and functional transfer is possible," Gene, vol. 503, no. 1, pp. 165-176, 2012.

[46] P. Kjellbom, C. Larsson, I. Johansson, M. Karlsson, and U. Johanson, "Aquaporins and water homeostasis in plants," Trends in Plant Science, vol. 4, no. 8, pp. 308-314, 1999.

[47] A. Bansal and R. Sankararamakrishnan, "Homology modeling of major intrinsic proteins in rice, maize and Arabidopsis: comparative analysis of transmembrane helix association and aromatic/arginine selectivity filters," BMC Structural Biology, vol. 7, article 27, 2007.

[48] J. Y. Jang, D. G. Kim, Y. O. Kim, J. S. Kim, and H. Kang, "An expression analysis of a gene family encoding plasma membrane aquaporins in response to abiotic stresses in Arabidopsis thaliana," Plant Molecular Biology, vol. 54, no. 5, pp. 713-725, 2004.

[49] R. Zardoya, "Phylogeny and evolution of the major intrinsic protein family, Biology of the Cell, vol. 97, no. 6, pp. 397-414, 2005. 
[50] F. Magni, C. Sarto, D. Ticozzi et al., "Proteomic knowledge of human aquaporins,” Proteomics, vol. 6, no. 20, pp. 5637-5649, 2006.

[51] E. Alexandersson, L. Fraysse, S. Sjövall-Larsen et al., "Whole gene family expression and drought stress regulation of aquaporins," Plant Molecular Biology, vol. 59, no. 3, pp. 469-484, 2005.

[52] C. Zhu, D. Schraut, W. Hartung, and A. R. Schäffner, "Differential responses of maize MIP genes to salt stress and ABA," Journal of Experimental Botany, vol. 56, no. 421, pp. 2971-2981, 2005.

[53] R. Aroca, G. Amodeo, S. Fernández-Illescas, E. M. Herman, F. Chaumont, and M. J. Chrispeels, "The role of aquaporins and membrane damage in chilling and hydrogen peroxide induced changes in the hydraulic conductance of maize roots," Plant Physiology, vol. 137, no. 1, pp. 341-353, 2005.

[54] N. Uehlein, K. Fileschi, M. Eckert, G. P. Bienert, A. Bertl, and R. Kaldenhoff, "Arbuscular mycorrhizal symbiosis and plant aquaporin expression," Phytochemistry, vol. 68, no. 1, pp. 122129, 2007.

[55] F. Lopez, A. Bousser, I. Sissoëff et al., "Diurnal regulation of water transport and aquaporin gene expression in maize roots: contribution of PIP2 proteins," Plant and Cell Physiology, vol. 44, no. 12, pp. 1384-1395, 2003.

[56] R. K. Vandeleur, G. Mayo, M. C. Shelden, M. Gilliham, B. N. Kaiser, and S. D. Tyerman, "The role of plasma membrane intrinsic protein aquaporins in water transport through roots: diurnal and drought stress responses reveal different strategies between isohydric and anisohydric cultivars of grapevine," Plant Physiology, vol. 149, no. 1, pp. 445-460, 2009.

[57] D. M. Gibeaut, J. Hulett, G. R. Cramer, and J. R. Seemann, "Maximal biomass of Arabidopsis thaliana using a simple, lowmaintenance hydroponic method and favorable environmental conditions," Plant Physiology, vol. 115, no. 2, pp. 317-319, 1997.

[58] I. S. Wallace, W.-G. Choi, and D. M. Roberts, "The structure, function and regulation of the nodulin 26-like intrinsic protein family of plant aquaglyceroporins," Biochimica et Biophysica Acta, vol. 1758, no. 8, pp. 1165-1175, 2006.

[59] F. Ishikawa, S. Suga, T. Uemura, M. H. Sato, and M. Maeshima, "Novel type aquaporin SIPs are mainly localized to the ER membrane and show cell-specific expression in Arabidopsis thaliana," FEBS Letters, vol. 579, no. 25, pp. 5814-5820, 2005.

[60] C. Maurel, F. Tacnet, J. Güclü, J. Guern, and P. Ripoche, "Purified vesicles of tobacco cell vacuolar and plasma membranes exhibit dramatically different water permeability and water channel activity," Proceedings of the National Academy of Sciences of the United States of America, vol. 94, no. 13, pp. 7103-7108, 1997.

[61] X. Yu, Y. H. Peng, M. H. Zhang, Y. J. Shao, W. A. Su, and Z. C. Tang, "Water relations and an expression analysis of plasma membrane intrinsic proteins in sensitive and tolerant rice during chilling and recovery," Cell Research, vol. 16, no. 6, pp. 599-608, 2006.

[62] L. Guo, Y. W. Zi, H. Lin et al., "Expression and functional analysis of the rice plasma-membrane intrinsic protein gene family," Cell Research, vol. 16, no. 3, pp. 277-286, 2006.

[63] V. Terzi, G. Pastori, P. R. Shewry, N. Di Fonzo, A. Michele Stanca, and P. Faccioli, "Real-time PCR-assisted selection of wheat plants transformed with HMW glutenin subunit genes," Journal of Cereal Science, vol. 41, no. 1, pp. 133-136, 2005.

[64] H. J. Schouten, F. A. Krens, and E. Jacobsen, "Do cisgenic plants warrant less stringent oversight?” Nature Biotechnology, vol. 24, no. 7, p. 753, 2006.
[65] S. G. Joshi, J. G. Schaart, R. Groenwold, E. Jacobsen, H. J. Schouten, and F. A. Krens, "Functional analysis and expression profiling of HcrVf1 and HcrVf2 for development of scab resistant cisgenic and intragenic apples," Plant Molecular Biology, vol. 75, no. 6, pp. 579-591, 2011.

[66] M. Katsuhara, Y. Akiyama, K. Koshio, M. Shibasaka, and K. Kasamo, "Functional analysis of water channels in barley roots," Plant and Cell Physiology, vol. 43, no. 8, pp. 885-893, 2002.

[67] M. Katsuhara, K. Koshio, M. Shibasaka, Y. Hayashi, T. Hayakawa, and K. Kasamo, "Over-expression of a barley aquaporin increased the shoot/root ratio and raised salt sensitivity in transgenic rice plants," Plant and Cell Physiology, vol. 44, no. 12, pp. 1378-1383, 2003.

[68] S. Mahajan and N. Tuteja, "Cold, salinity and drought stresses: an overview," Archives of Biochemistry and Biophysics, vol. 444, no. 2, pp. 139-158, 2005.

[69] G.-T. Huang, S.-L. Ma, L.-P. Bai et al., "Signal transduction during cold, salt, and drought stresses in plants," Molecular Biology Reports, vol. 39, no. 2, pp. 969-987, 2012.

[70] J. Y. Jang, S. H. Lee, J. Y. Rhee, G. C. Chung, S. J. Ahn, and H. Kang, "Transgenic Arabidopsis and tobacco plants overexpressing an aquaporin respond differently to various abiotic stresses," Plant Molecular Biology, vol. 64, no. 6, pp. 621632, 2007.

[71] C. Tournaire-Roux, M. Sutka, H. Javot et al., "Cytosolic pH regulates root water transport during anoxic stress through gating of aquaporins," Nature, vol. 425, no. 6956, pp. 393-397, 2003.

[72] R. Gu, X. Chen, Y. Zhou, and L. Yuan, "Isolation and characterization of three maize aquaporin genes, ZmNIP2;1, ZmNIP2;4 and ZmTIP4;4 involved in urea transport," BMB Reports, vol. 45, no. 2, pp. 96-101, 2012.

[73] T. Horie, T. Kaneko, G. Sugimoto et al., "Mechanisms of water transport mediated by PIP aquaporins and their regulation via phosphorylation events under salinity stress in barley roots," Plant and Cell Physiology, vol. 52, no. 4, pp. 663-675, 2011.

[74] T. Matsumoto, H.-L. Lian, W.-A. Su et al., "Role of the aquaporin PIP1 subfamily in the chilling tolerance of rice," Plant and Cell Physiology, vol. 50, no. 2, pp. 216-229, 2009.

[75] H.-L. Lian, X. Yu, D. Lane, W.-N. Sun, Z.-C. Tang, and W.-A. Su, "Upland rice and lowland rice exhibited different PIP expression under water deficit and ABA treatment," Cell Research, vol. 16, no. 7, pp. 651-660, 2006. 

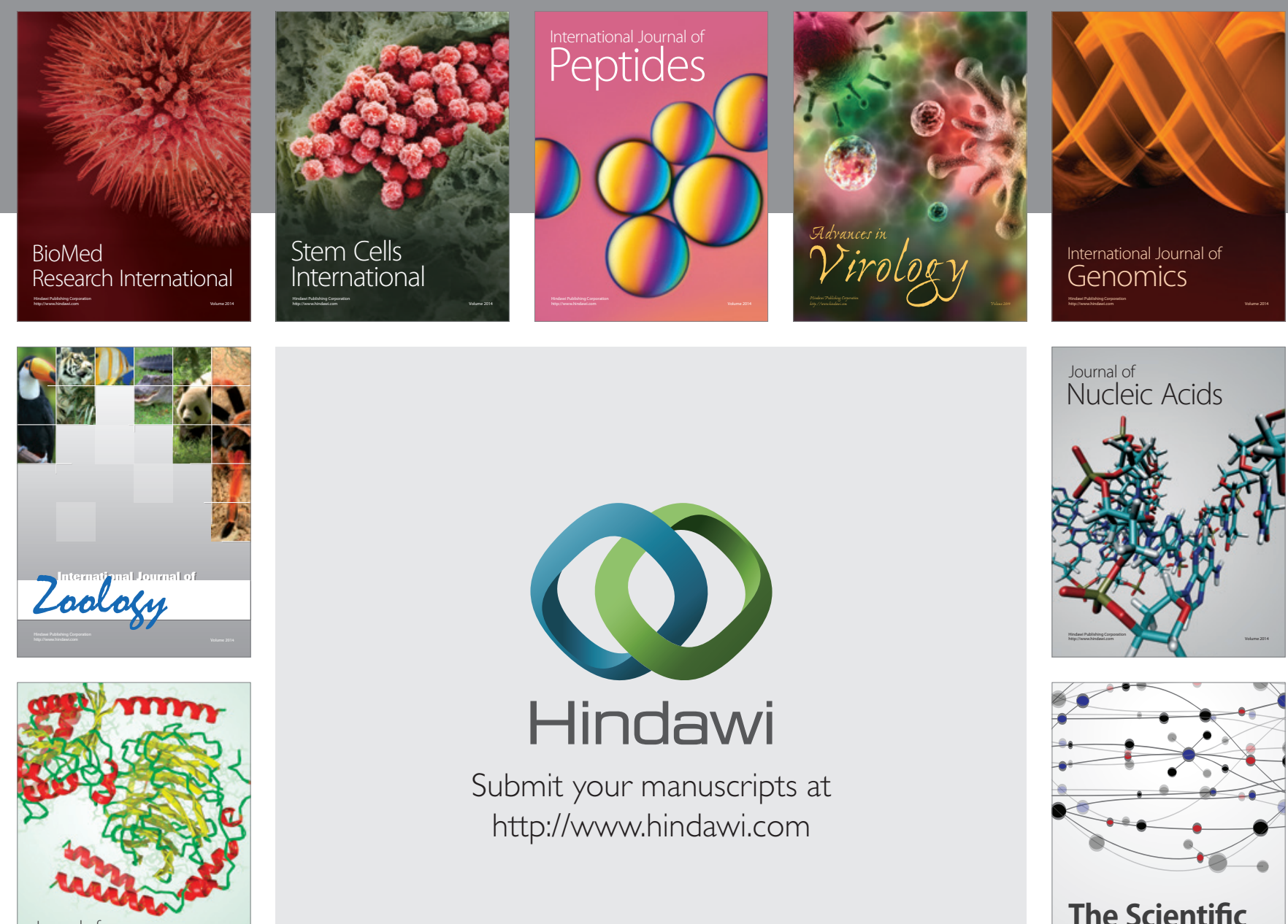

Submit your manuscripts at

http://www.hindawi.com

Journal of
Signal Transduction
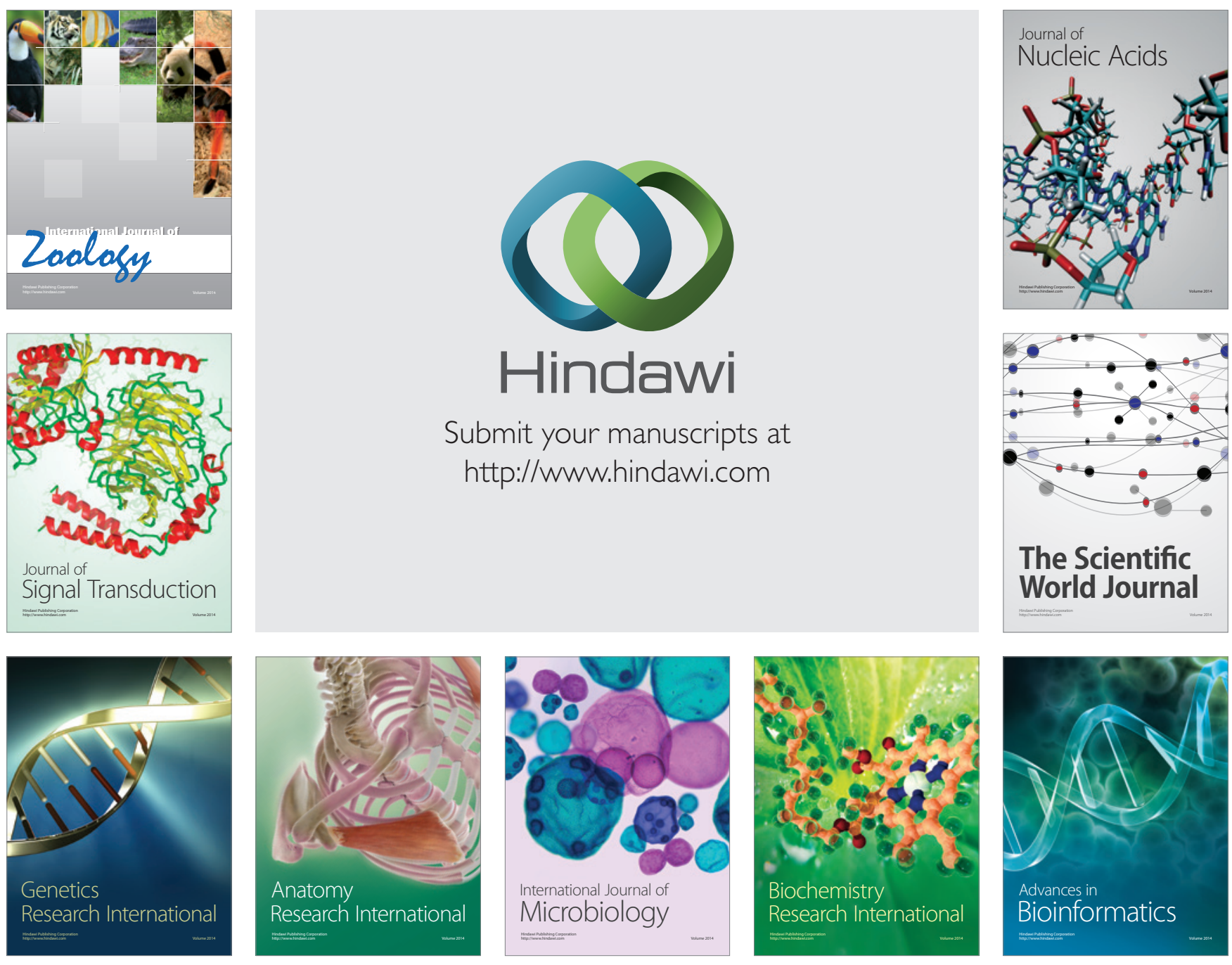

The Scientific World Journal
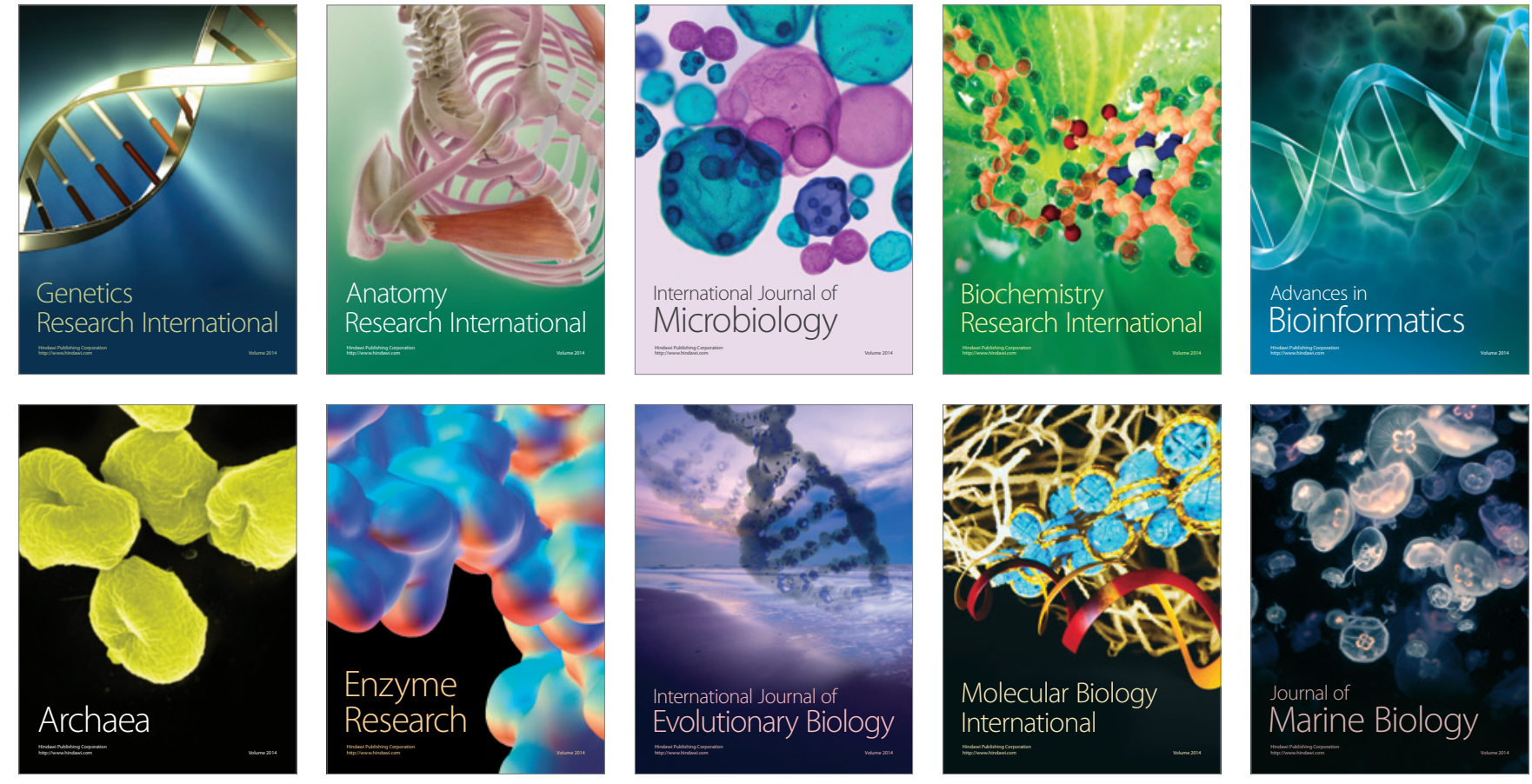